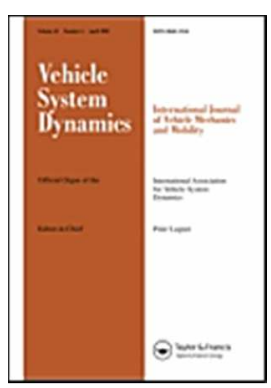

\title{
Lap time simulation and design optimisation of a brushed DC electric motorcycle for the Isle of Man TT Zero Challenge
}

\begin{tabular}{|r|l|}
\hline Journal: & Vehicle System Dynamics \\
\hline Manuscript ID & NVSD-2016-0393.R2 \\
\hline Manuscript Type: & Original Paper \\
\hline Date Submitted by the Author: & 29-May-2017 \\
\hline Complete List of Authors: & $\begin{array}{l}\text { Dal Bianco, Nicola; University of Padova, Mechanical Engineering } \\
\text { Lot, Roberto; University of Southampton, Department of Mechanical } \\
\text { Engineering; University of Padova, Department of Industrial Engineering } \\
\text { Matthys, Koen; Brunel University }\end{array}$ \\
\hline Keywords: & $\begin{array}{l}\text { Electric vehicles, Motorcycle < Bicycle, Optimal design < Ergonomic, } \\
\text { Optimal control < Control, Optimization }\end{array}$ \\
\hline
\end{tabular}


To appear in Vehicle System Dynamics Vol. 00, No. 00, Month 20XX, 1-29

\title{
Lap time simulation and design optimisation of a brushed DC electric motorcycle for the Isle of Man TT Zero Challenge
}

\section{Introduction}

\begin{abstract}
This works regards the design of an electric motorcycle for the annual Isle of Man TT Zero Challenge. Optimal control theory was used to perform lap time simulation and design optimisation. A bespoked model was developed, featuring 3D road topology, vehicle dynamics and electric power train, composed of a lithium battery pack, brushed DC motors and motor controller. The model runs simulations over the entire $37.73 \mathrm{mi}$ or $60 \mathrm{~km}$ of the Snaefell Mountain Course. The work is validated using experimental data from the BX chassis of the Brunel Racing team, which ran during the 2009 to 2015 TT Zero races. Optimal control is used to improve drive train and power train configurations. Findings demonstrate computational efficiency, good lap time prediction and design optimisation potential, achieving a 2 minute reduction of the reference lap time through changes in final drive gear ratio, battery pack size and motor configuration.
\end{abstract}

Keywords: Electric vehicles, motorcycle, desing, optimisation, optimal control,

Electric (EV) and hybrid electric (HEV) vehicles are continuously gaining in popularity, as the automotive industry is responding to tough environmental regulatory challenges and is aiming to meet growing consumer demand for energy consumption reduction and more sustainable living.

Contemporary with the wider adoption of electric road vehicles, motorsports too has seen an electrification movement. More driven by the innovation aspect and opportunities through technological advantage, it brought forward consideration for low carbon racing categories. The pinnacle of motorsport, Formula 1, opened up regulations to introduce hybridization in 2009. Energy-efficiency competitions have gained widespread appeal such as the Shell Eco Marathon, while an all new FIA electric racing single seater championship Formula E saw the light in 2014. Motorcycle racing led the way however, with TTXGP holding the first international low carbon, zero-emission Grand Prix on the Isle of Man in 2009. TTXGP was succeeded by the TT Zero Challenge and incorporated as an official event class of the historic Isle of Man Tourist Trophy or TT from 2010, where racing has been taking place annually on the infamous $37.73 \mathrm{mi}$ Snaefell Mountain course since 1911.

\footnotetext{
* Corresponding author. Email: roberto.lot@soton.ac.uk
} 
Generally speaking, the energy consumption and performance of EVs depend significantly on driving inputs and vehicle design parameters, therefore optimisation techniques have been applied to EVs as demonstrated in literature [13, 19, 29, 32, 34, 38, 39]. For road vehicles, where the performance is not of primary importance, optimisation is mainly related to energy management, while for racing vehicles the main goal is minimum time optimisation $[1,22,27]$.

TT Zero is a time trial race whereby electric motorcycles aim to complete one $60 \mathrm{~km}$ lap on closed public roads along the Snaefell Mountain Course. Achieving minimum time is thus the main goal, and the optimisation of such a motorcycle would fall into the racing category. However, energy management optimisation is not negligible due to the unusual length of the TT course, therefore the TT Zero Challenge fits in-between a road and race application and corresponding design considerations must be made.

This work aims to optimise through optimal control lap time simulations a TT Zero motorcycle design, as run by the Brunel Racing team from Brunel University London. Brunel Racing has entered an electric racing motorcycle in the inaugural TTXGP and in every subsequent TT Zero Challenge to date. Experimental data gathered during testing and racing on the Isle of Man will serve to validate the optimal control model presented here. Using an optimal control approach for the TT Zero application is a challenging proposition for two main reasons: first, the length of the Mountain Course is nearly 12 times that of a common racing circuit; second, the mathematical model must capture both the vehicle dynamics and power-train characteristics.

Optimal control simulations have been used in recent years for lap time optimisation of various vehicles, but to the best of the authors' knowledge, such work has not yet been reported on for applications comparable in complexity to that of the TT Zero Challenge. Examples of optimal control lap time simulations for car models can be found in literature $[7-9,17,18,20,22,25,26,28,36,37]$. Among those studies, only one however deals with a hybrid vehicle [22]. For motorcycles models, fewer examples of optimal control work exist $[2,10-12,33]$, and none of them regard an electric motorcycle. Optimisation studies related to electric vehicles have thus far been performed with other strategies than optimal control simulations [13, 19, 31, 32, 38, 39], even though optimal control theory could be applied, as already demonstrated by [22, 29, 34].

Our in-house developed model presented here does for the first time offer a comprehensive lap time and design optimisation for TT Zero, by incorporating a motorcycle dynamics model, an electric power train configuration model and a race course over a long distance with three-dimensional track geometry including non-negligible slope and elevation changes.

Using a sound modelling approach for all relevant characteristics of motorcycle, power train and road is key to successfully solve the optimal control problem. Indeed, the complexity of the motorcycle and power train models, together with the long road that increases the numerical size of the problem, may lead to long computational solving times or even to a non-solvable problem. In this work, we therefore adopt an indirect strategy $[4,6]$ and a particular numerical solver [2] to solve the optimal control problem. The indirect approach does require knowledge of the exact equations of the optimal control problem, and therefore an analytical model for the motorcycle, power train and road geometry has to be provided. For this reason, commercial multibody software tools like ADAMS, Virtual Lab Motion, CarSim, RecurDyn, SimPack, etc. are of limited use here.

In the next section, the motorcycle, power train and road models are described in further detail. The mathematical models introduced aim to correctly capture real world behaviour, while remaining simple enough to allow computations to run in just a few minutes. In the section that follows after that, simulation outcomes are validated through 
comparison with experimental data from the TT Zero race. Finally, the models are used in a design optimisation exercise, focusing on three critical performance parameters for the electric motorcycle: direct drive gear ratio, battery pack size and motor configuration.

\section{Model}

\subsection{Road}

The TT course runs for $37.73 \mathrm{mi}$ or approximately $60 \mathrm{~km}$ over closed public roads and the Snaefell Mountain on the Isle of Man (British Isles). The road is characterised by several slopes and elevation variations, thus a three-dimensional model of the track is required in order to perform realistic simulations. The approach used to describe the track and the vehicle position along the circuit is similar to the one described in earlier work [24]. The road, shown in figure 1 , is modeled as a ribbon which is described by its middle lane position $\boldsymbol{C}(s)$ relative to an absolute frame:

$$
\boldsymbol{C}(s)=\left(x_{t}(s), y_{t}(s), z_{t}(s)\right)^{T}
$$

plus the orientation matrix $\boldsymbol{T}(s)$ of the ribbon, which is given by three consecutive rotations about the Cartesian axes:

$$
\boldsymbol{T}(s)=R_{z}(\theta) R_{y}(\sigma) R_{x}(\xi)
$$

where $R_{i}(\nu)$ is the rotation of an angle $\nu$ about the $i-t h$ cartesian axis $(x, y$ or $z)$. $C(s)$ can be expressed as function of the curvilinear abscissa $s$ (i.e. the arc length along the road centre line) by integrating in $s$ the first column of the rotation matrix $\boldsymbol{T}(s)$, as will be described later in this section. The angles $\theta, \sigma$ and $\xi$ are respectively the road heading (i.e. the direction of traveling), slope (i.e. traveling uphill or downhill) and banking (i.e. the road leaning). In this work the banking angle has not been considered (i.e. $\xi=0$ ) due to lack of data about the actual road geometry. The road position $\boldsymbol{C}(s)$ and orientation

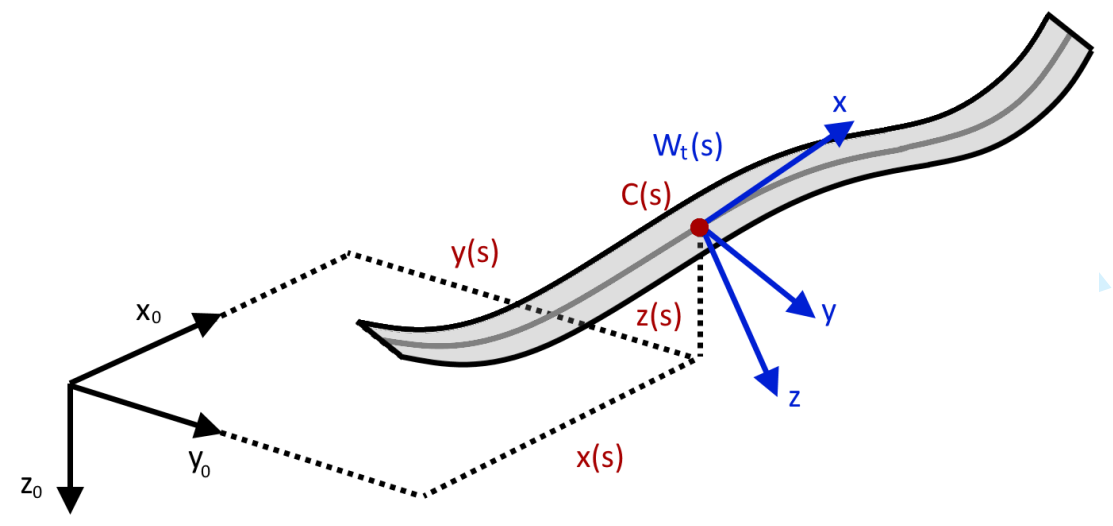

Figure 1.: Coordinate system of the strip-road model.

$\boldsymbol{T}(s)$ can be put together into a $4 \times 4$ matrix $\boldsymbol{W}_{t}$ that represents the road reference 
system:

$$
\boldsymbol{W}_{t}(s)=\left(\begin{array}{cccc}
\cos (\theta) \cos (\sigma) & -\sin (\theta) & \cos (\theta) \sin (\sigma) & x \\
\sin (\theta) \cos (\sigma) & \cos (\theta) & \sin (\theta) \sin (\sigma) & y \\
-\sin (\sigma) & 0 & \cos (\sigma) & z \\
0 & 0 & 0 & 1
\end{array}\right)
$$

where the upper left three dimensional submatrix is the orientation matrix, and the right-most column is the translation vector (D-H convention, [14]). Finally, the width $r_{w}(s)$ completes the road characterisation.

It is thus necessary to know the road geometry $\boldsymbol{W}_{t}(s)$ and width $r_{w}(s)$ in order to perform any simulation on the TT course. Such information can be obtained from Google satellite images, which give a flat representation of the road, combined with a tool that provides elevation data. As described in [21] the data so obtained is affected by a certain noise (both due to intrinsic data error and to manual selection of the road boundary points) that needs to be eliminated in order to improve the road accuracy and for numerical reasons. One possible way to reduce the noise is through a least square optimal control problem [21], using the following procedure. First, a set of points lying on the road's left and right borders are hand-selected using Google satellite images, and the coordinates (latitude and longitude) of such points are stored; the number of points to be selected should be enough to capture the relevant road geometry variations. Thus, a two-dimensional map of the course is obtained, but the information about the road elevation is still missing. The road elevation can be obtained from databases containing the Earth surface elevation data; an online tool www.gpsvisualizer.com allows to extract the elevation from a list of point coordinates. Combining Google satellite photos with elevation data, two sets of points in the 3D space belonging respectively to the road's left $x_{l 0}, y_{l 0}, z_{l 0}$ and right $x_{r 0}, y_{r 0}, z_{r 0}$ borders are obtained as function of an estimate $\zeta$ of the road curvilinear abscissa $s$ (i.e. the arc length of the estimated road center). The road data thus obtained is shown in figure 2; the green markers indicate the value of the estimated curvilinear abscissa $\zeta$ every 10 kilometers. A least square optimal control problem is then used to find the road curvilinear abscissa $s$ and the road reference system $\boldsymbol{W}_{t}(s)(3)$ that makes the resulting road to best match the set of the acquired $3 \mathrm{D}$ points, satisfying at the same time a certain smoothness requirement. This optimal control problem is formulated as follows:

$$
\underset{u_{\theta}, u_{\sigma}, u_{w}, u_{s}}{\operatorname{minimize}} \int_{\zeta_{0}}^{\zeta_{f}} S(\boldsymbol{x})+w_{u} U\left(\boldsymbol{u}_{t}\right) \quad d \zeta
$$

subject to:

$$
\begin{aligned}
& \frac{d \mathbf{x}_{t}}{d \zeta}=\mathbf{g}\left(\boldsymbol{x}_{t}, \boldsymbol{u}_{t}\right) \\
& s(0)=0
\end{aligned}
$$

where $\boldsymbol{x}$ and $\boldsymbol{u}$ are respectively the state variable and control vectors:

$$
\begin{aligned}
& \mathbf{u}_{t}=\left(u_{\theta}, u_{\sigma}, u_{w}, u_{s}\right)^{T} \\
& \mathbf{x}_{t}=\left(x_{t}, y_{t}, z_{t}, \theta, \sigma, \hat{\theta}, \hat{\sigma}, r_{w}, s\right)^{T}
\end{aligned}
$$



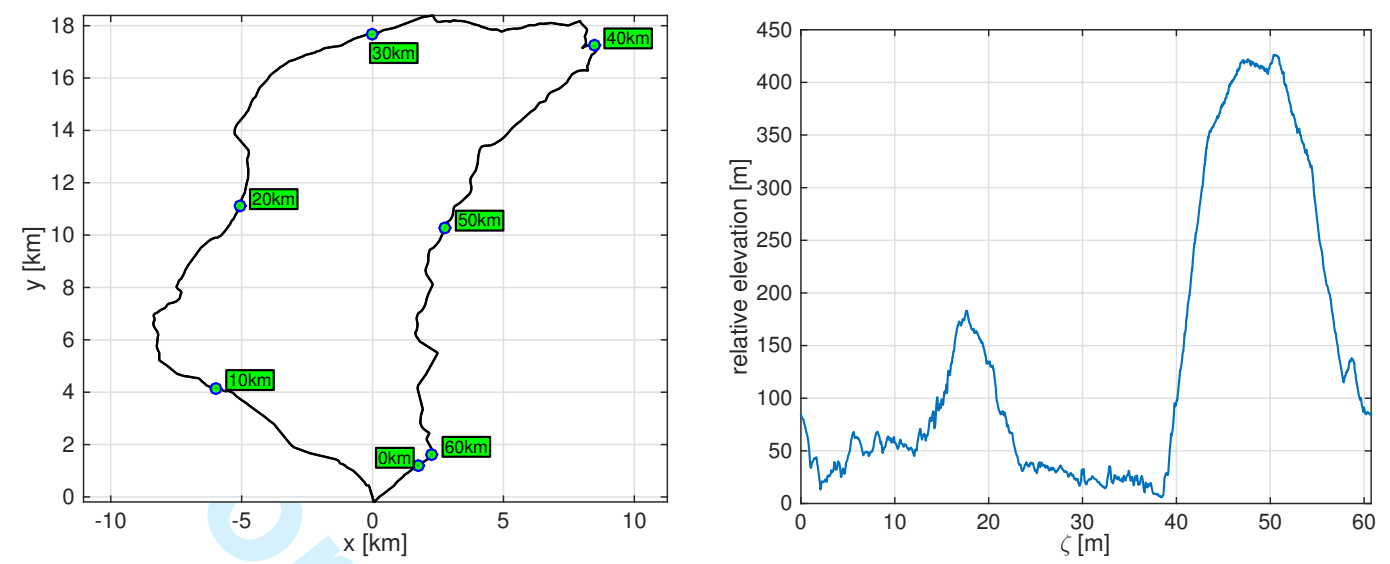

Figure 2.: Illustration of the Snaefell Mountain Course road data. The $x-y$ coordinates (left image) have been acquired from Google satellite images, while the elevation profile was derived via an online tool www.gpsvisualizer.com. In the left picture the $x-y$ view is represented. The green markers indicate the value of the estimated curvilinear abscissa $\zeta$ every 10 kilometers. The right one shows the relative elevation of the TT course. The elevation peak in correspondence of the Snaefell Mountain is evident.

The target to minimize (4a) is the sum of two terms: the first $S(\boldsymbol{x})$ is the distance of the road borders from the measured ones which makes the resulting road fit the acquired data, while the second $U(\boldsymbol{u})$ is a control penalty term that makes the road smooth:

$$
\begin{aligned}
S\left(\boldsymbol{x}_{t}\right)= & \left(\frac{x_{r}-x_{r 0}}{\sigma_{e}}\right)^{2}+\left(\frac{y_{r}-y_{r 0}}{\sigma_{e}}\right)^{2}+\left(\frac{z_{r}-z_{r 0}}{\sigma_{e}}\right)^{2} \\
& +\left(\frac{x_{l}-x_{l 0}}{\sigma_{e}}\right)^{2}+\left(\frac{y_{l}-y_{l 0}}{\sigma_{e}}\right)^{2}+\left(\frac{z_{l}-z_{l 0}}{\sigma_{e}}\right)^{2} \\
U\left(\boldsymbol{u}_{t}\right)= & u_{\theta}^{2}+u_{\sigma}^{2}+u_{w}^{2}+u_{s}^{2}
\end{aligned}
$$

where $x_{r}, y_{r}, z_{r}$ (respectively $x_{l}, y_{l}, z_{l}$ ) are the coordinates of the right road border (respectively left). These coordinates can be easily expressed as function of the state variables $\boldsymbol{x}$ :

$$
\begin{aligned}
& x_{r}=x_{t}-\frac{1}{2} r_{w} \sin (\theta) \quad y_{r}=y_{t}+\frac{1}{2} r_{w} \cos (\theta) \quad z_{r}=z_{t} \\
& x_{l}=x_{t}+\frac{1}{2} r_{w} \sin (\theta) \quad y_{l}=y_{t}-\frac{1}{2} r_{w} \cos (\theta) \quad z_{l}=z_{t}
\end{aligned}
$$

The target $U\left(\boldsymbol{u}_{t}\right)$ in (6b) makes the road satisfy a certain smoothness because the controls $\boldsymbol{u}_{t}$ are related to the second derivatives of the road angles with respect to $\zeta$. Indeed, the 
explicit first order equations (4b) are:

$$
\begin{aligned}
& \frac{d \theta}{d \zeta}=\hat{\theta}\left(1+u_{s}\right) \quad \frac{d \sigma}{d \zeta}=\hat{\sigma}\left(1+u_{s}\right) \\
& \frac{d \hat{\theta}}{d \zeta}=u_{\theta} \quad \frac{d \hat{\sigma}}{d \zeta}=u_{\sigma} \\
& \frac{d r_{w}}{d \zeta}=u_{w} \\
& \frac{d x_{t}}{d \zeta}=\cos (\sigma) \cos (\theta)\left(1+u_{s}\right) \quad \frac{d y_{t}}{d \zeta}=\cos (\sigma) \sin (\theta)\left(1+u_{s}\right) \quad \frac{d z_{t}}{d \zeta}=-\sin (\sigma)\left(1+u_{s}\right) \\
& \frac{d s}{d \zeta}=\left(1+u_{s}\right)
\end{aligned}
$$

Equations (8a) and (8b) relate the controls to the second order derivative of the road angles $\theta$ and $\sigma$, while equation (8d) expresses the variation of $\boldsymbol{C}$ as function of $\boldsymbol{T}$, and finally equation (8e) describes how the road curvilinear abscissa $s$ differs from the estimated one $\zeta$.

The solution of the above described optimal control problem gives as output the road position $\boldsymbol{C}(s)$ and orientation $\boldsymbol{T}(s)$ as a function of the curvilinear abscissa $s$. Figure 3 focuses on a small part of the course comprised between $40.95 \mathrm{~km}<s<41.4 \mathrm{~km}$, and shows that the resulting road borders (blue lines) match the input points (red crosses) well. At the same time, the road elevation, the $x-y$ curvature $\kappa$ and the slope demonstrate smooth signal profiles.
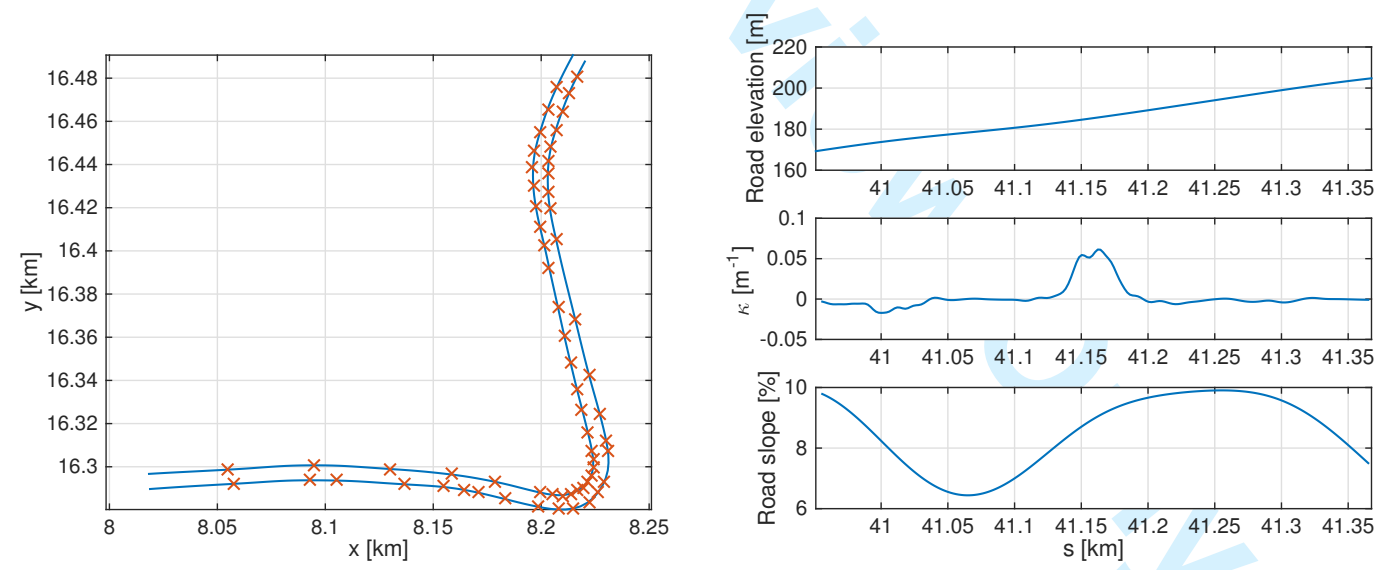

Figure 3.: The road geometry obtained through solving the optimal control problem is shown here for a small part of the course in the range $40.95 \mathrm{~km}<s<41.4 \mathrm{~km}$. The left image shows the road input data (red crosses) and borders resulting from the optimal control problem (blue lines) for the part of road comprised between $40.95 \mathrm{~km}<s<$ $41.4 \mathrm{~km}$. Road elevation, $x-y$ curvature $\kappa$ and slope percentage are shown in the figure one the right. 


\section{2. $\quad$ Motorcycle model}

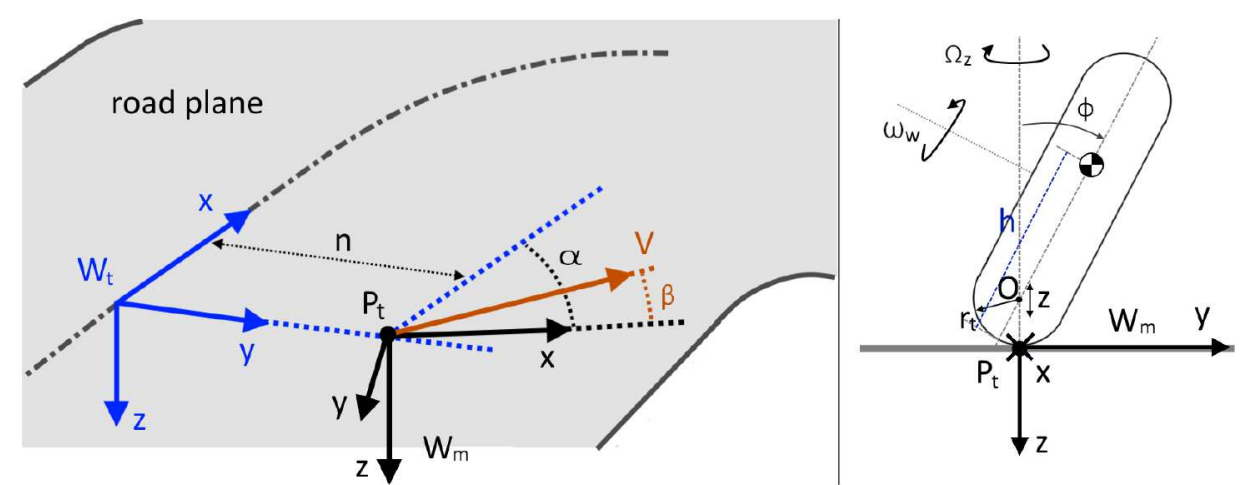

Figure 4.: In the left image, an illustration of the vehicle frame $\mathbf{W}_{m}$ with respect to the road frame $\mathbf{W}_{t}$. The relative heading $\alpha$ and the drift angle $\beta$ are shown. In the right image, the mono-wheel is shown with respect the frame $\mathbf{W}_{m}$. The yaw rate $\Omega_{z}$, the roll angle $\phi$, the vertical displacement $z$ and the wheel spin $\omega_{w}$ are highlighted.

Since the TT Mountain Course is approximately $60 \mathrm{~km}$ long, a simple model is required to execute optimal control simulations over such a long distance in a reasonable amount of time. Indeed, the use of a motorcycle model with several degrees of freedom over such a long road can make the resulting optimal control problem difficult to solve, leading to long (e.g. hours) computational times or even to a numerically non solvable problem $[4,6]$. The simplest way to describe a vehicle running while staying in contact with the road is a single body that moves on the road plane; however, for a motorcycle the roll motion is very important because it has a remarkable influence on the dynamics. Therefore, the most simple model that captures the essence of the motorcycle dynamics (i.e. g-g diagram and roll motion) is a body that can move and roll on the road plane, plus a rotating body which accounts for the gyroscopic effects. Such bodies can be thought of as a mono-wheel moving on the road plane, where its mass and inertia tensor are those of the motorcycle.

The motorcycle position and orientation on the road plane are described by means of three variables: the curvilinear abscissa along the road middle line $s$, the lateral displacement from the centre line $n$ and the relative heading to road centre line direction $\alpha$ (see figure 4). The frame $W_{m}$, which is attached to the motorcycle and its origin coincides with the mono-wheel tyre contact point, is thus generated from the frame $\boldsymbol{W}_{t}(3)$ by a lateral displacement of a quantity $n$, followed by a rotation $\alpha$ on the road plane:

$$
W_{m}=W_{t}(s)\left(\begin{array}{cccc}
\cos (\alpha) & -\sin (\alpha) & 0 & 0 \\
\sin (\alpha) & \cos (\alpha) & 0 & n \\
0 & 0 & 1 & 0 \\
0 & 0 & 0 & 1
\end{array}\right)
$$

The motorcycle, together with the frame $\boldsymbol{W}_{m}$, moves on the road surface with speed $V$, sideslip angle $\beta$ and yaw rate $\Omega_{z}$ (see figure 4). Moreover, the mono-wheel is free to translate vertically (the vertical displacement is $z$ ), to roll by an angle $\phi$ and to spin with angular velocity $\omega_{w}$ (positive when the motorcycle moves forward), for a total of six degrees of freedom. The speed $V$ lies in the road surface, the vertical displacement $z$, 
together with the yaw rate $\Omega_{z}$, is aligned with the $z$-axis of the frame $\boldsymbol{W}_{m}$, i.e. they are perpendicular to the road plane. The roll rotation is about an axis parallel to the $x$-axis of the frame $\boldsymbol{W}_{m}$, but passing through the center of the wheel toroid (the point $O$ in figure 4), so the contact point between the wheel and the tarmac is always in the origin of $\boldsymbol{W}_{m}$. Finally, the wheel spin $\omega_{w}$ is about the wheel center.

The time variation of the tracking variables $s, n$ and $\alpha$ can be expressed in terms of the speed variables $V, \beta, \Omega_{z}$, and of the road curvature in the $x-y$ transversal plane $\kappa$ by the following kinematic relationships:

$$
\begin{aligned}
& \dot{s}=\frac{V(\beta \sin \alpha+\cos \alpha)}{1-n \kappa} \\
& \dot{n}=V \sin \alpha-V \beta \cos \alpha \\
& \dot{\alpha}=\Omega_{z}-\kappa \frac{V \cos \alpha+V \beta \sin \alpha}{1-n \kappa}
\end{aligned}
$$

Since the sideslip angle $\beta$ is generally smaller than $10^{\circ}$, the approximations $\sin (\beta) \approx \beta$ and $\cos (\beta) \approx 1$ have been adopted. Equations 10 track the vehicle position and orientation relative to the road by simply integrating the vehicle speed, drift angle and yaw rate together with road curvature, so the vehicle longitudinal $s$ and lateral position $n$ and relative heading $\alpha$ are immediately available as state variables. This is a significant advantage in comparison to the Cartesian coordinate approach, which requires separate algorithms to estimate such information.

For the derivation of the motorcycle equations of motion (relative to $V, \beta, \phi, z, \omega_{w}$ ) in the frame $\boldsymbol{W}_{m}$, it is useful to give the expression of the angular velocity $\boldsymbol{\Omega}_{m}=$ $\left(\Omega_{x}, \Omega_{y}, \Omega_{z}\right)^{T}$ and of velocity $\boldsymbol{V}_{m}=\left(v_{x}, v_{y}, v_{z}\right)^{T}$ of the frame $\boldsymbol{W}_{m}$. All of them can be expressed as a function of the state variables and of road geometry:

$$
\begin{gathered}
\boldsymbol{\Omega}_{m}=\left(\begin{array}{c}
\Omega_{x} \\
\Omega_{y} \\
\Omega_{z}
\end{array}\right)=\left(\begin{array}{c}
\dot{s}(\nu \sin \alpha+\tau \cos \alpha) \\
\dot{s}(\nu \cos \alpha-\tau \sin \alpha) \\
\Omega_{z}
\end{array}\right) \\
\boldsymbol{V}_{m}=\left(\begin{array}{c}
v_{x} \\
v_{y} \\
v_{z}
\end{array}\right)=\left(\begin{array}{c}
V \\
-V \beta \\
\dot{s} \tau n
\end{array}\right)
\end{gathered}
$$

where $\nu$ and $\tau$ are respectively the road curvature in the sagittal plane $x-z$ and the torsion. We point out that the velocity $\boldsymbol{V}_{m}$ and the angular velocity $\boldsymbol{\Omega}$ of the frame $\boldsymbol{W}_{m}$ in (11) can be fully expressed as function of state variables by substituting $\dot{s}$ in (11) with expression (10a). Moreover road curvatures $\kappa, \nu$ in (11) and torsion $\tau$ in (10) can be expressed in terms of the road orientation angles [24]:

$$
\begin{aligned}
& \tau=-\sin (\sigma) \theta^{\prime} \\
& \nu=\sigma^{\prime} \\
& \kappa=\cos (\sigma) \theta^{\prime}
\end{aligned}
$$

where the superscript ' indicates the derivative with respect to the curvilinear abscissa $s$.

The Newton and Euler equations of motion for the mono-wheel model have been derived using MBSymba [23], a Maple library for the automatic generation of the equations 
of motion of multibody systems with a symbolic approach. The Newton equations along the three axis of the frame $W_{m}$ have which describe the time evolution of $V, \beta, z$, are:

$$
\begin{array}{r}
M \dot{v}_{x}+\left[\cos \phi\left(-h+r_{t}\right)-r_{t}+z\right] M \dot{\Omega}_{y}-2 M\left(h-r_{t}\right)\left(\Omega_{z} \cos \phi-\Omega_{y} \sin \phi\right) \dot{\phi} \\
-M \sin \phi\left(h-r_{t}\right) \dot{\Omega}_{z}+2 M \Omega_{y} \dot{z}-M \Omega_{x} \Omega_{z}\left(h-r_{t}\right) \cos \phi+M \Omega_{x} \Omega_{y}\left(h-r_{t}\right) \sin \phi \\
+M\left[\left(-r_{t}+z\right) \Omega_{x}+v_{y}\right] \Omega_{z}+M \Omega_{y} v_{z}=F_{x}-D-M g \sigma \cos \alpha \\
M \dot{v}_{y}+M \ddot{\phi} \cos (\phi)\left(h-r_{t}\right)+M\left[\left(h-r_{t}\right) \cos \phi+r_{t}-z\right] \dot{\Omega}_{x}-M \sin \phi\left(h-r_{t}\right) \dot{\phi}^{2} \\
-2 M \sin \phi \Omega_{x}\left(h-r_{t}\right) \dot{\phi}-2 M \Omega_{x} \dot{z}-M\left(h-r_{t}\right)\left(\Omega_{z}^{2}+\Omega_{x}^{2}\right) \sin \phi \\
-M \Omega_{y} \Omega_{z}\left(h-r_{t}\right) \cos \phi-M\left[\left(r_{t}-z\right) \Omega_{y}-v_{x}\right] \Omega_{z}-M \Omega_{x} v_{z} \\
=F_{y}+L \sin \phi+M g \sigma \sin \alpha \\
+M \sin \phi\left(h-r_{t}\right) \dot{\Omega}_{x}+M \dot{v}_{z}+M \cos \phi\left(h-r_{t}\right)\left(\Omega_{x}^{2}+\Omega_{y}^{2}\right)+M \Omega_{y} \Omega_{z}\left(h-r_{t}\right) \sin \phi \\
-M\left(-r_{t}+z\right) \Omega_{x}^{2}+M \Omega_{x} v_{y}-M\left(-r_{t}+z\right) \Omega_{y}^{2}-M \Omega_{y}=M g-N-L \cos \phi \\
M v_{x} \sin (\phi)\left(h-r_{t}\right)+M \cos \phi\left(h-r_{t}\right) \dot{\phi}^{2}+2 M \cos \phi \Omega_{x}\left(h-r_{t}\right) \dot{\phi}
\end{array}
$$

where $M$ is the total mass of the motorcycle plus the rider, $h$ is the reference distance of the center of mass $(\mathrm{CoM})$ from ground, $r_{t}$ is the wheel cross section radius, $F_{x}, F_{y}$ and $N$ are respectively the wheel longitudinal, lateral and normal force, $D$ is the drag force, $L$ is the aerodynamic lift force. Since the road slope $\sigma$ is always smaller than $10^{\circ}$, the approximations $\sin \sigma \approx \sigma$ and $\cos \sigma \approx 1$ have been adopted. Moreover, the Euler equation governing the roll dynamics, calculated with respect to the origin of the frame $\boldsymbol{W}_{m}$, is the following:

$$
\begin{array}{r}
\left\{\left[-\left(h-r_{t}\right)\left(z-r_{t}\right) \cos \phi+\left(h-r_{t}\right)^{2}\right] M+I_{x x}\right\} \ddot{\phi}+I_{x x} \dot{\Omega}_{x} \\
+\left(\Omega_{z} \cos \phi-\Omega_{y} \sin \phi\right) I_{g y} \omega_{w}+\sin \phi\left(z-r_{t}\right) L \\
+\left(I_{y y}-I_{z z}\right)\left[\cos \phi \sin \phi\left(\Omega_{y}^{2}-\Omega_{z}^{2}\right)+\Omega_{y} \Omega_{z}\left(1-2 \cos \phi^{2}\right)\right]+M T_{\phi}=0
\end{array}
$$

where $I_{x x}, I_{y y}, I_{z z}$ are the inertia moments of the motorcycle with respect to the Cartesian axes, $I_{g y}=2 I_{w y}+I_{e y} \gamma_{c}$ is the gyroscopic moment of inertia, i.e. the sum of the front and rear wheel y-axis inertia moment $I_{w y}$ and the engine y-axis inertia $I_{e y}$, taking into account the motor to wheel gear ratio $\gamma_{c} . T_{\phi}$ is the sum of several terms proportional to the mass of the mono-wheel which appears as consequence that the Euler equation is not written with respect to the center of gravity. The chosen pole (the origin of the frame $\boldsymbol{W}_{m}$ ) has the advantage that tyre forces does not appear in equation (14). The 
expression of $T_{\phi}$ is the following:

$$
\begin{aligned}
T_{\phi}= & \left(h-r_{t}\right)\left[\sin \phi\left(\ddot{z}-\Omega_{y} v_{x}+\Omega_{x} v_{y}+\dot{v}_{z}-g\right)\right. \\
& \left.-\cos \phi\left(\dot{v}_{y}-2 \Omega_{x} \dot{z}+\Omega_{x} v_{z}-\Omega_{z} v_{x}+g \sigma \sin \alpha\right)\right] \\
& +\left(h-r_{t}\right)\left(r_{t}-z\right)\left[2 \cos \phi \dot{\Omega}_{x}-2 \cos \phi \Omega_{z} \Omega_{y}+\sin \phi\left(\Omega_{y}^{2}-\Omega_{z}^{2}-\dot{\phi}^{2}-2 \Omega_{x} \dot{\phi}\right)\right] \\
& +\left(h-r_{t}\right)^{2}\left[\dot{\Omega}_{x}+\sin \phi \cos \phi\left(\Omega_{y}^{2}-\Omega_{z}^{2}\right)-2 \cos ^{2} \phi \Omega_{x} \Omega_{y}\right] \\
& +\left(z^{2}-2 r_{t} z\right) \dot{\Omega}_{x}+(h-z)\left(h-2 r_{t}+z\right) \Omega_{y} \Omega_{z} \\
& +\left(r_{t}-z\right)\left(\dot{v}_{y}-v_{z} \Omega_{x}+v_{x} \Omega_{z}-2 \dot{z} \Omega_{x}-g \sigma \sin \alpha\right)
\end{aligned}
$$

Another equation is required to describe the spin motion of the mono-wheel, which is the Euler equation of the mono-wheel about its y-axis:

$$
\begin{aligned}
& \dot{\omega}_{w} I_{w y}=\tau_{w}-\tau_{r}-F_{x}\left[r-r_{t}(1-\cos \phi)-z \cos \phi\right] \\
& +I_{w y}\left[\dot{\Omega}_{y} \cos \phi+\sin \phi \dot{\Omega}_{z}+\left(\Omega_{z} \cos \phi-\Omega_{y} \sin \phi\right) \dot{\phi}\right]
\end{aligned}
$$

where $\tau_{w}$ is the driving torque applied to the rear wheel, $\tau_{r}$ is the rolling resistance torque and $I_{w y}$ is the inertia moment of the wheel only. The six variables used in equations (13), (14), (16) to capture the six degrees of freedom of the mono-wheel are: $V, \beta, z, \phi, \Omega_{z}, \omega_{w}$. No equation is used to describe the time evolution of the yaw rate $\Omega_{z}$ because it is a control input.

The previous equations depend on the external forces and torques, and on the internal driving torque $\tau_{w}$ acting on the motorcycle. The driving torque $\tau_{w}$ is actually the sum of the positive traction motor torque and the negative braking torque:

$$
\tau_{w}=\tau_{t}+\tau_{b} \Longrightarrow \tau_{t}=f^{+}\left(\tau_{w}\right), \quad \tau_{b}=f^{-}\left(\tau_{w}\right)
$$

where $f^{+}(x)$ and $f^{-}(x)$ are two functions that return the positive and negative part of the argument respectively; the simultaneous usage of the brake and the gas is not allowed in this model. The external forces are the tyre longitudinal force $F_{x}$, the tyre lateral force $F_{y}$, and the aerodynamic $\operatorname{drag} D$ and lift $L$; the only external torque is the rolling resistance $\tau_{r}$. The tyre longitudinal $F_{x}$ and lateral $F_{y}$ forces, together with the rolling resistance $\tau_{r}$ and the tyre load $N$ are given by a linear tyre model, limited by an adherence ellipse:

$$
\begin{aligned}
F_{x} & =N K_{\kappa} \kappa_{w} \\
F_{y} & =N\left(K_{\beta} \beta+K_{\phi} \phi\right) \\
\tau_{r} & =\left(N \cos \phi+F_{y} \sin \phi\right) \mu_{r} \\
N & =f^{+}\left(K_{s} z+K_{c} \dot{z}\right)
\end{aligned}
$$

where $K_{\kappa}, K_{\beta}$ and $K_{\phi}$ are the tyre longitudinal, lateral and roll stiffness, $K_{s}$ and $K_{c}$ are the tyre radial stiffness and damping stiffness, and finally the function $f^{+}$ensures the tyre load to be non negative. The tyre forces $F_{x}$ and $F_{y}$ are forced to lie within the tyre 
adherence ellipse:

$$
\left(\frac{F_{x}}{N \mu_{x}}\right)^{2}+\left(\frac{F_{y}}{N \mu_{y}}\right)^{2} \leq 1
$$

where $\mu_{x}$ and $\mu_{y}$ are the the maximum longitudinal and lateral tyre adherence espectively. The longitudinal tyre slip, $\kappa_{w}$, is given by:

$$
\kappa_{w}=\frac{\omega_{w}\left[r-r_{t}+\cos \phi\left(r_{t}-z\right)\right]-V}{V}
$$

where $r$ is the rear wheel radius. Finally, the drag and lift aerodynamic forces are proportional to the square of the motorcycle speed:

$$
\begin{aligned}
D & =\frac{1}{2} \rho_{a} C_{d} V^{2} \\
L & =\frac{1}{2} \rho_{a} C_{l} V^{2}
\end{aligned}
$$

where $\rho_{a}$ is the air density, $C_{d}$ and $C_{l}$ are the drag and lift coefficients. The numeric values of the motorcycle data used to feed the model are listed in appendix A.

\subsection{Powertrain model}

For the inaugural TTXGP grand prix in 2009, a wide variety of first designs were brought to the grid. The BX electric motorcycle chassis from the Brunel Racing team carried a simple power train, consisting of a single brushed DC motor, matching controller and a Lithium Ion battery pack. From 2010 and the first TT Zero Challenge onward, the BX power train was upgraded to a dual brushed DC motor configuration, matching controller and various sizes of a Lithium Polymer battery pack. This configuration became a favourite among entries for TTXGP national championships and Isle of Man TT Zero events, and best represents first generation designs. The BX dual motor power train with Li-Po pack from Brunel Racing was developed year on year until the 2015 TT Zero event, and it is this latest version BX-15 that has been modelled here.

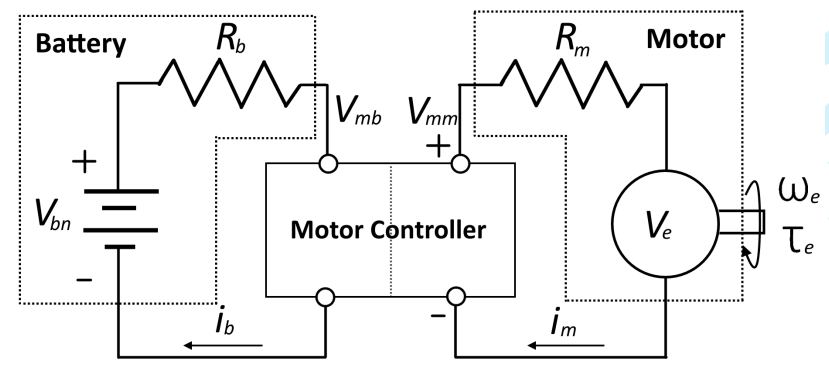

Figure 5.: Equivalent electric circuits of battery and electric motor.

The BX-15 power train is composed of two customized Agni 95R brushed DC and axial flux motors, supplied by a bespoke Kokam Li-Po battery pack in "4P24S" (4 parallel of 24 series) cell arrangement and governed by an off-the-shelf $1200 A$ Kelly KDHE controller. 
The two motors have been balanced so that the total power delivery is equally split over each one. The electrical equivalent model used to describe the behaviour of such an electric power train is given in figure 5 and is composed of two main circuits, a battery circuit and a motor circuit, connected via a motor controller that drives the motor voltage/current input so as to deliver the requested torque at the motor output shaft.

The two motors in the power train have been modelled via a single motor circuit: indeed, two motors working in parallel can be seen as equivalent to a single motor of the same characteristics but half the internal resistance. The battery pack is modelled via a battery circuit, composed of an ideal voltage supply $V_{b n}$ in series with a resistor $R_{b}$ that accounts for the battery pack's internal resistance [15]. Output voltage Vbn decreases linearly with battery discharge status, from $V_{b n 1}$ when the battery pack is fully charged to $V_{b n 0}$ when it is fully discharged. Therefore, the closed loop battery voltage $V_{m b}$ may be calculated as follows:

$$
\begin{aligned}
& V_{m b}=V_{b n}-i_{b} R_{b} \\
& V_{b n}=V_{b n 0}+\left(V_{b n 1}-V_{b n 0}\right) \frac{e}{e_{i}}
\end{aligned}
$$

where $i_{b}$ is the current flowing in the batteries, $e$ and $e_{i}$ are respectively the actual and initial battery charge status. The motor controller is a DC to DC converter from the battery circuit to the motor circuit, and for simplicity the conversion efficiency $\eta_{m c}$ is assumed to be constant, yielding to the following power balance equation:

$$
V_{m m} i_{m}=\eta_{m c} V_{m b} i_{b}
$$

where $V_{m m}$ is the motor controller voltage output to the motor circuit which is related to the current $i_{m}$ circulating in the motor and the the self-induced or back electromagnetic force (e.m.f.) $V_{e}$. Indeed, in steady condition the voltage balance is:

$$
V_{m m}=i_{m} R_{m}(T)+V_{e}
$$

where the $R_{m}$ represents the armature winding resistance, which depends on motor temperature $T$. Finally, the conversion of the electric power into mechanical power is governed by the well known proportional equations [15, 16]:

$$
\begin{array}{r}
V_{e}=\kappa_{V}(T) \omega_{m} \\
\tau_{e}=\kappa_{\tau}(T) f^{+}\left(i_{m}-i_{m 0}\right)
\end{array}
$$

where $\omega_{m}$ is the motor rotational speed, $i_{m 0}$ is the engine current offset, $\tau_{e}$ the motor torque, $\kappa_{\tau}(T)$ the torque constant and $\kappa_{V}(T)$ the back e.m.f constant, with $\kappa_{\tau}(T)<$ $\kappa_{V}(T)$ because of eddy current and other conversion losses.

In the equations (24) and (25) three motor characteristics depend on the motor temperature: the armature winding resistance $R_{m}$ and the just mentioned proportional constants $\kappa_{\tau}, \kappa_{V}$. Specifically, $R_{m}$ increases with motor temperature while $\kappa_{\tau}(T), \kappa_{V}(T)$ diminish because the hotter the motor, the lower the magnetic flux of the permanent magnet is. A linear relationship has been used to capture the variation with temperature of these 
three quantities:

$$
\begin{array}{r}
R_{m}(T)=R_{m 0}+\sigma_{R}\left(T-T_{0}\right) \\
\kappa_{V}(T)=\kappa_{V 0}-\sigma_{m}\left(T-T_{0}\right) \\
\kappa_{\tau}(T)=\kappa_{\tau 0}-\sigma_{m}\left(T-T_{0}\right)
\end{array}
$$

where $T_{0}$ is the corresponing temperature for the reference values $R_{m 0}, \kappa_{V 0}$ and $\kappa_{\tau 0}$.

Since motor characteristics vary with motor temperature, another equation is required to describe the motor temperature variation over time. The motor heat balance allows to calculate the motor temperature rate on the basis of incoming $Q_{\text {in }}$ and outgoing $Q_{\text {out }}$ heat flux:

$$
C \dot{T}=Q_{\text {in }}-Q_{\text {out }}
$$

where

$$
\begin{aligned}
Q_{\text {in }} & =V_{m m} i_{m}-\omega_{m} \tau_{e} \\
Q_{\text {out }} & =q\left(T-T_{\text {ext }}\right)
\end{aligned}
$$

where $C$ is the motor thermal capacity, $T_{\text {ext }}$ is the environmental temperature and $q$ is the conduction coefficient, which is asusmed to vary linearly from $q_{0}$ when the motorcycle is stopped, to $q_{1}$ at a speed $V_{1}=150 \mathrm{~km} / \mathrm{h}$ :

$$
q=q_{0}+\frac{q_{1}-q_{0}}{V_{1}} V
$$

It is worth pointing out that no dynamic effects have been included in the model of the electric power train: indeed, electromagnetic transients are much faster than mechanical ones and the former may be neglected for our purposes. In other words, for a step variation of the controlled voltage $V_{m m}$ (or current, $i_{m}$ ) it is reasonable to assume that the current $i_{m}$ (or voltage, $V_{m m}$ ) as well as the motor torque $\tau_{e}$ vary instantaneously, while the variation of the motor speed $\omega_{m}$ and e.m.f. $V_{e}$ are governed by vehicle inertia through equation (16).

In conclusion using equations (24) and (25), the controller voltage $V_{m m}$ can be expressed as function of the motor torque $\tau_{e}$ and motor speed $\omega_{e}$

$$
V_{m m}=V_{e}+i_{m} R_{m}=\omega_{e} \kappa_{V}+\frac{\tau_{e}}{\kappa_{\tau}} R_{m}
$$

Moreover, taking into account the powertrain efficiency $\eta_{c}$ and the gear ratio $\gamma_{c}$ between the motor speed $\omega_{e}$ and wheel speed $\omega\left(\omega_{e}=\omega_{w} \gamma_{c}\right)$, it is possible to express $\tau_{e}$ as a function of the wheel traction torque $\tau_{t}$ :

$$
\tau_{e}=\frac{\tau_{t}}{\eta_{c} \gamma_{c}}
$$

Using equations (22) and (23) it is also possible to obtain an expression for the battery current $i_{b}$ and motor controller voltage at the battery side $V_{m b}$ as function of the motor 
torque $\tau_{e}$ and speed $\omega_{e}$

$$
\begin{aligned}
i_{b} & =\frac{V_{b n}}{2 R_{b}}-\frac{\sqrt{\eta_{m}\left(V_{b n}^{2} \eta_{m}-4\left(\frac{\tau_{e}}{\kappa_{\tau}}\right)^{2} R_{b} R_{m}-4 \frac{\tau_{e}}{\kappa_{\tau}} R_{b} \omega_{e} \kappa_{V}\right)}}{2 R_{b} \eta_{m}} \\
V_{m b} & =V_{b n}-i_{b} R_{b}
\end{aligned}
$$

The expression of $i_{b}$ is useful because it allows to evaluate the discharge rate of the battery charge:

$$
\dot{e}=-V_{b n} i_{b}
$$

Also, efficiency values both of the motor only and of the power train i.e. motor, battery pack and motor controller, are shown in figure 6 as a function of motor speed and motor torque. It can be noticed that the brushed DC motors used in BX-15 are characterised by an efficiency up to $\approx 85 \%$, and that further losses in the complete power train drop the efficiency value by an additional $\approx 5 \%$.
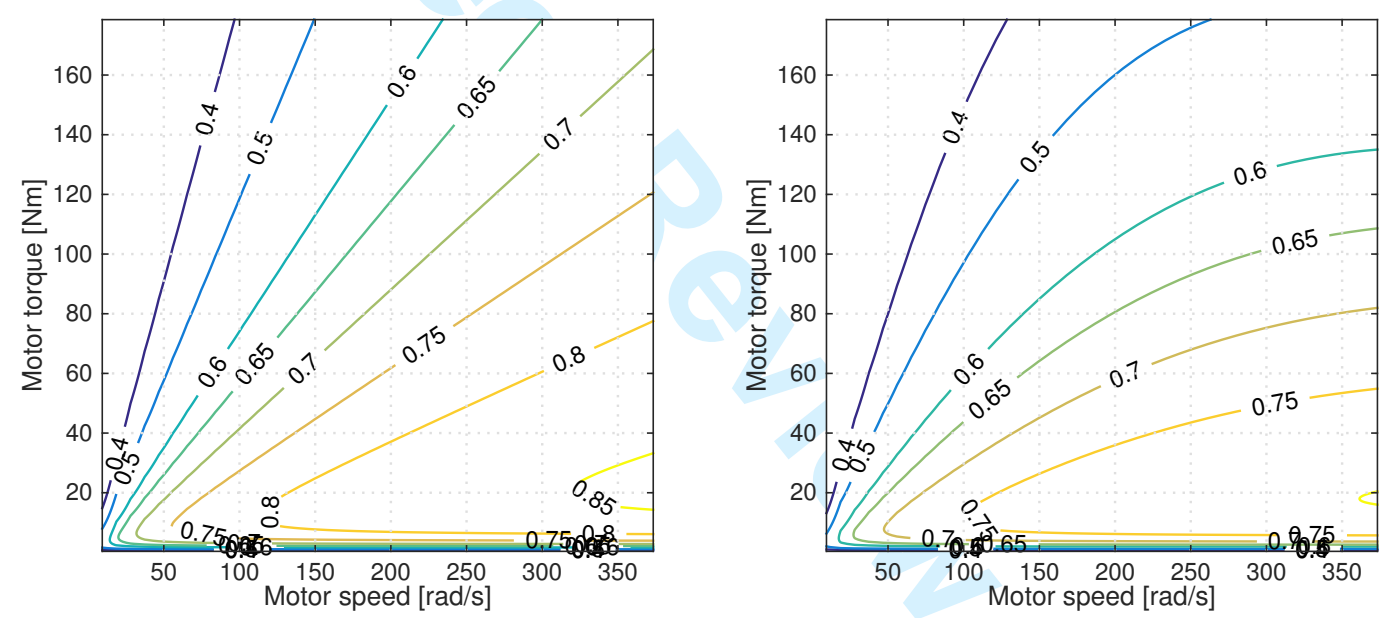

Figure 6.: Contour plot of efficiency values as a function of the motor speed and motor torque. The left plot refers to the motor efficiency only, while the right plot shows overall power train efficiency, including motor, battery pack and motor controller. Data shown was derived for a motor temperature of $25^{\circ} \mathrm{C}$.

Finally, we recall here that the only variables that the electric motor model adds to the state space model are the motor temperature $T$ and the battery charge $e$. All other quantities are algebraic expressions of other variables and controls.

\subsection{State space formulation}

The vehicle dynamics and electric power train equations described in the previous sections can be put together to form a first order ordinary differential equation system that completely characterises the model. In order to do this, two auxiliary variables have to be introduced because both roll angle $\phi$ and the vertical displacement $z$ appear respectively 
in equation (14) and (13) with the second order derivative:

$$
\begin{aligned}
& \dot{\phi}=\omega_{\phi} \\
& \dot{z}=z_{d o t}
\end{aligned}
$$

At this point, equations (10), (13), (14), (16), (27), (33), (34) completely describe the mono-wheel dynamics as a system of 12 first order differential equations with as many state variables $\mathbf{x}_{s}$ and 2 inputs $\mathbf{u}_{s}$ :

$$
\begin{aligned}
& \mathbf{x}_{s}=\left\{s, n, \alpha, V, \beta, z, \phi, \omega_{w}, T, e, \omega_{\phi}, z_{d o t}\right\}^{T} \\
& \mathbf{u}_{s}=\left\{\Omega_{z}, \tau_{w}\right\}
\end{aligned}
$$

Recalling that the motor torque $\tau_{e}$ can be easily expressed as function of the control $\tau_{w}(31)$, all other electric variables as $V_{m m}, i_{m}, i_{b}, V_{b}$ can be expressed as function of $\tau_{w}$ and the state variable $\omega_{w}$ using equations (25) (24) (32). Using the state variables and controls expressed in (35) and the equations (10), (13), (14), (16), (27), (33), (34), the complete model can be described by a system of first order differential equations that depends on the control $\boldsymbol{u}_{s}$ :

$$
\boldsymbol{A}_{s}\left(\boldsymbol{x}_{s}\right) \dot{\boldsymbol{x}}_{\boldsymbol{s}}=\boldsymbol{f}\left(\boldsymbol{x}_{s}, \boldsymbol{u}_{s}\right)
$$

where matrix $\boldsymbol{A}_{s}$ is invertible provided that $V>0$, i.e. the vehicle never stops, and $n<1 / \kappa(s)$, i.e. the vehicle never passes over the local curvature centre of the road.

\section{Optimal control problem formulation}

The minimum time problem is formulated here as an optimal control problem with the same approach presented in [26], [25], [24], and will be briefly summarized here. The Optimal Control Problem (OCP) consists in finding the vehicle control inputs $\boldsymbol{u}_{s}$ that drive the vehicle from the starting grid line $s_{0}$ to the end $s_{f}$ of the TT course in the minimum time, while satisfying a set of differential equality and algebraic inequality constraints. For this reason, as described in [24], it is convenient to change the the independent variable from $t$ to $s$ in the equations of motion (36). Such variable change is based on the following derivation rule:

$$
\dot{\boldsymbol{x}_{s}}=\frac{d \boldsymbol{x}_{s}}{d t}=\frac{d \boldsymbol{x}_{s}}{d s} \frac{d s}{d t}=\boldsymbol{x}_{s}^{\prime} \dot{s}
$$

Using this transformation the optimal control problem can be formulated in the curvilinear abscissa domain:

$$
\begin{aligned}
\text { find: } & \min _{\boldsymbol{u} \in \boldsymbol{U}} t=\int_{s_{0}}^{s_{f}} \frac{1}{\dot{s}} d s \\
\text { subject to: } & \boldsymbol{A}_{s} \boldsymbol{x} \prime=\boldsymbol{f}(\boldsymbol{x}, \boldsymbol{u}, s) \\
& \boldsymbol{\psi}(\boldsymbol{x}, \boldsymbol{u}, s) \leq \mathbf{0} \\
& \boldsymbol{b}\left(\boldsymbol{x}\left(s_{0}\right), \boldsymbol{x}\left(s_{f}\right)\right)=\mathbf{0}
\end{aligned}
$$


where (38b) is the state space model (i.e. the equations of motion (36) transformed using $(37)),(38 \mathrm{c})$ are a set of algebraic inequalities involving both the state variables and control inputs and (38d) is the set of boundary conditions used to specify the vehicle state at the beginning and at the end of the manoeuvre. In this work the vehicle initial state $\boldsymbol{x}(0)$ has been fixed, while the final one has been left free.

In the specific case of the mono-wheel model, unilateral constraints equations (38c) are used to make the simulation withstand the real physical constraints; in particular, they ensure that:

(a) the motorcycle never exceeds the road borders, considering that the track has width $r_{w}(s)$

(b) the roll rate is lower than the maximum one real driver can handle $\omega_{\phi}^{\max }$;

(c) the yaw rate is less than the one real motorcycle can achieve $\Omega_{z}^{\max }$ (this contraint has to be included because the yaw rate is a control);

(d) the tyre forces never exceed the adherence ellipse, as already discussed in 2 (the maximum longitudinal and lateral tyre adherence are $D_{x}, D_{y}$ respectively);

(e) the motor current remains lower than the maximum motor current $i_{m}^{\max }$;

(f) the power absorbed by the motor controller from the battery pack is lower than the maximum battery pack power $P_{b}^{\max }$;

(g) the battery charge $e$ does not become negative.

Such conditions can be expressed by the following relationships:

$$
\begin{array}{r}
r_{w} / 2 \leq n \leq r_{w} / 2 \\
-\omega_{\phi}^{\text {max }} \leq \omega_{\phi} \leq \omega_{\phi}^{\max } \\
-\Omega_{z}^{\text {max }} \leq \Omega_{z} \leq \Omega_{z}^{\max } \\
\left(\frac{S}{N D_{x}}\right)^{2}+\left(\frac{S}{N D_{y}}\right)^{2} \leq 1 \\
i_{m} \leq i_{m}^{\max } \\
V_{b n} i_{b} \leq P_{b}^{\max } \\
e \geq 0
\end{array}
$$

The optimal control problem has been formulated and solved using a software suite [5] (https://github.com/mechatronix/TS-OCS) that is made of a Maple package for equation generation (called XOptima), a $C++$ library for numerical solution (called Mechatronix) and an embedded Ruby interpreter (called PINS). The effectiveness of this software suite, based on an optimal control indirect solution [30] [6] as described in [3], has been widely demonstrated in literature [25] [26] [5] [35] [36] [12] [10].

\section{Simulation results and model validation}

The minimum time optimal control simulation has been carried out on the entire Snaefell Mountain Course obtaining a theoretical lap time of 29 minutes and 31 seconds, which is in good agreement with the physical lap of 29 minutes and 47 seconds, registered during a practice session of the 2015 TT Zero event. The simulation took less than three minutes to compute on a desktop PC equipped with an Intel Core ir processor, using 


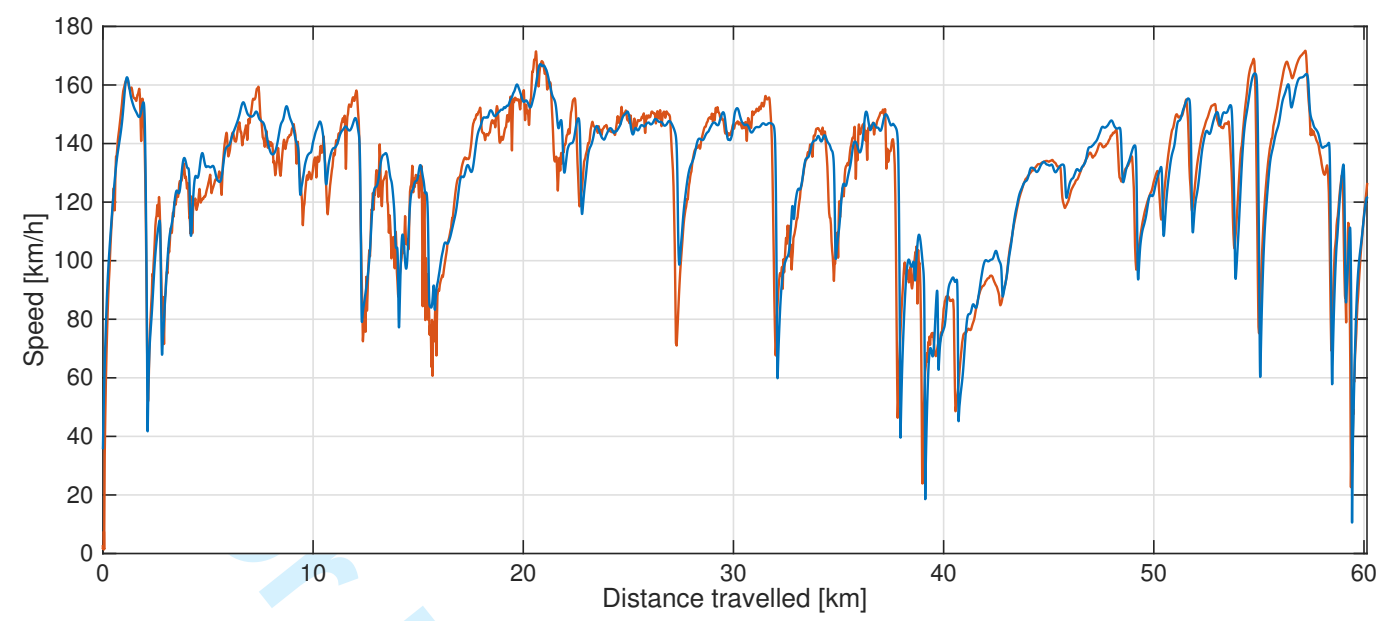

Figure 7.: Experimental (red) and simulated (blue) speed profile versus distance travelled.
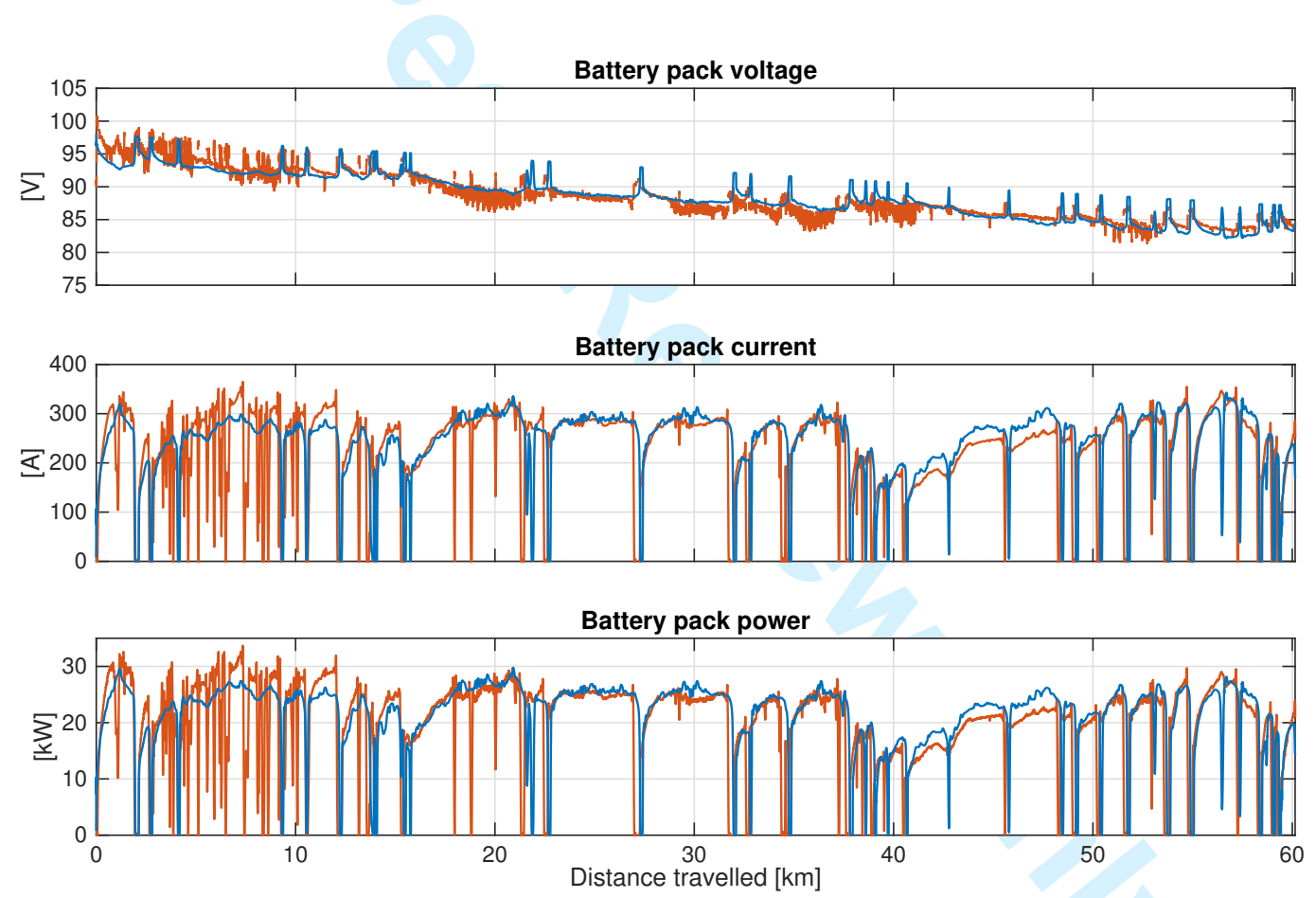

Figure 8.: Battery pack voltage (top graph), current (centre graph) and power (bottom graph) vs. distance travelled. Red lines refer to experimental data, blue lines to simulation.

a discretisation mesh with one point every meter (leading to a total of $\approx 60 e 3$ mesh points); this suggest that the used optimal control solver, together with the motorcycle model presented in this work, is very efficient. The simulated motorcycle speed along the track is shown in figure 7 together with the experimental one; the figure highlights a good qualitative agreement between the experimental data and the simulation. It can be observed that the simulation is slightly faster than the actual rider between the $40^{\text {th }}$ and 
Figure 9.: Motor voltage (top graph), current (centre graph) and power consumption (bottom graph) vs. distance travelled. Red lines refer to experimental data, blue lines to simulation.
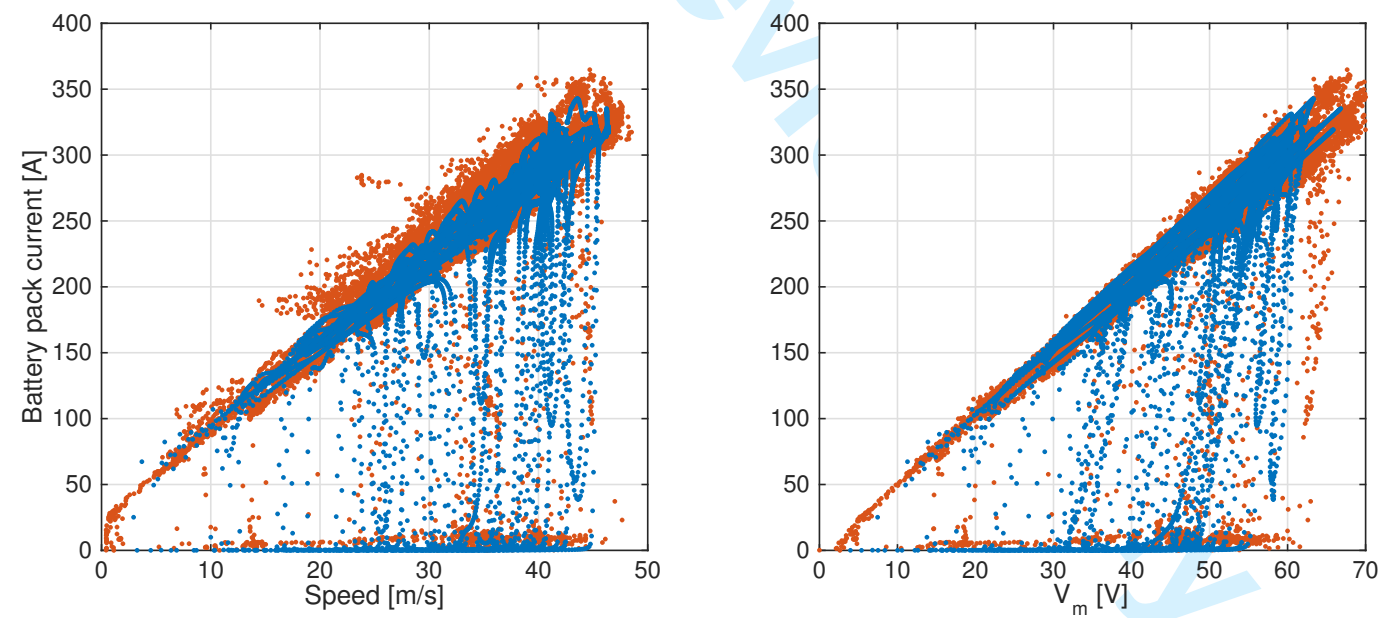

Figure 10.: Battery pack current draw vs. motorcycle speed (left) and motor driving voltage (right). The simulated data (blue dots) shows the same linear trend as the experimental data (red dots).

$50^{\text {th }}$ kilometre. This is the section of the course with the most significant positive slope (ascent of the Snaefell Mountain) and, as the bottom graph of figure 8 suggests, demands more power in simulation. As the real rider had some freedom not to apply maximum power over the entire course so as to prevent excessive motor load and preserve battery 


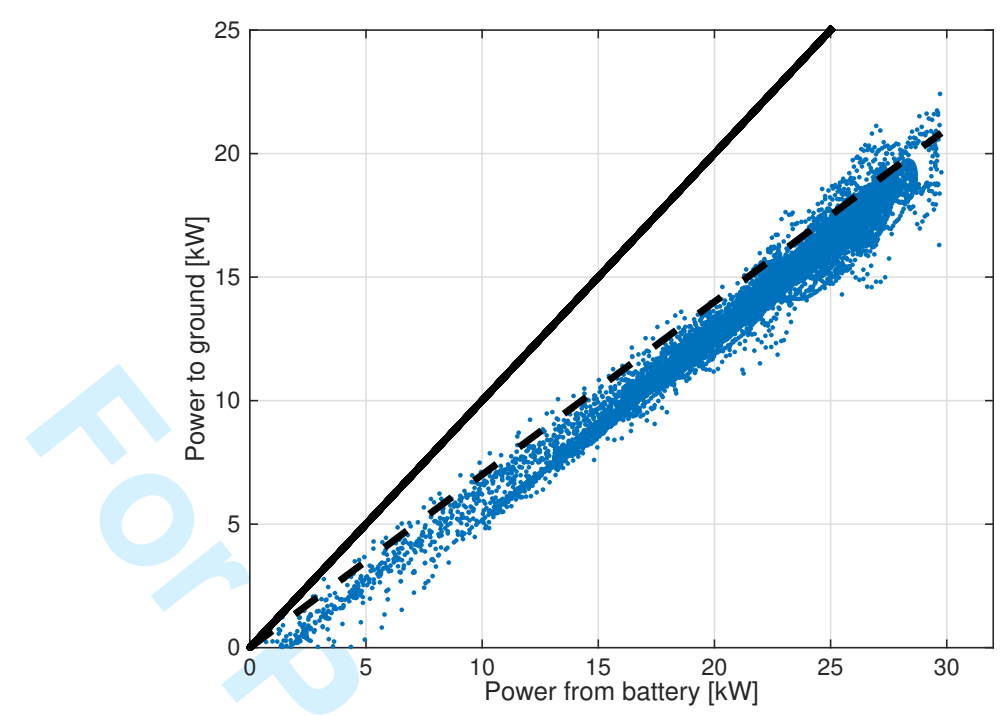

Figure 11.: Power delivered to the ground versus power drained from battery (simulated data). The black solid and dashed lines correspond respectively to an overall efficiency of the $100 \%$ and $70 \%$ : the simulated motorcycle highlights an efficiency close to the $70 \%$.

pack energy (even if battery pack capacity was sized with contingency to avoid full battery discharge), this may be the reason why the rider navigated the final climbing section of the course somewhat slower than he could, as optimal control simulation indicates.

Figure 8 reports on the energy usage along the course: battery pack voltage, current and power delivery in simulation are shown together with the experimental data, and demonstrate very good agreement for all three parameters. The simulated battery pack voltage (top graph) agrees well with the experimental data, capturing not only the expected decreasing trend of the battery discharge process but also the location and duration of voltage recovery effects when load is being removed. These voltage effects align with the current and power drops observed in the centre and bottom graphs respectively, as the rider releases the throttle. The simulated battery pack current draw and power drain further match well with current and power as measured on track, as well as with the speed graphs from figure 7, only showing slightly greater values for the first 15 kilometres of the course and slightly lower values between the $40^{\text {th }}$ and $50^{\text {th }}$ kilometre, indicating that the rider was pushing beyond optimal energy drain at the start and started to preserve energy near the end. It can be expected that there are additional smaller effects impacting on power drain and efficiency near the start and towards the final heavy load stages of the race, such as the cold start and high final temperatures, and the fact that battery charge status is always slightly above nominal in the beginning and close to non-linear drop-off for individual battery cells near the final stages. Even though the experimental data could not provide reliable specific temperature nor individual battery cell information to quantify the impact of such factors, the fact that the deviations observed between simulation and experiment in figure 8 occur only near the start and end of the race distance, exactly when both the rider would be adjusting his riding style and the aforementioned temperature and individual battery characteristics are most dominant, underlines that the modelling approach is sound.

Figure 9 shows the motor related variables. The simulated motor current (centre graph) however presents some differences with the experimental data. In the first part of the 
TT course, the experimental motor current is, on average, higher than the simulated current, while in the second part the opposite can be observed. The same behaviour can be noticed for the power absorbed by the motor, which is a consequence of the current deviations as power is calculated via the product of motor voltage and motor current. This discrepancy can be due to the uncertainty in the measurement of motor current: indeed, the motorcycle was equipped with two motors working in parallel, yet only the current drawn by the left motor has been captured due to a failure in the right motor sensor. Total motor current had to be estimated by multiplying the left current by two, as the dual motor configuration had been balanced at the start to have the two motors working equally. However, not only is it known that each brushed DC Agni $95 \mathrm{R}$ motor is subject to a degree of variation from the nominal specification due to the non-automated production process applied (affecting armature internal resistance for example), balancing of multiple motor configurations also has to performed track side, especially after servicing that must occur after each track outing. The balancing process consists of manual tuning (i.e. not via motor controller programming) of the brush holder set-up of each motor in the configuration, and is therefore prone to slight deviations around the ideal brush holder positions, which carries no impact for normal road and short circuit race applications but could affect individual motor temperature, power and efficiency, and therefore motor balancing within a multiple configuration, when under sustained heavy loading over a long distance such as around the TT Snaefell Mountain Course. And indeed, the two motors used here did turn out to have an asymmetric loading along the course, as found from temperature observations. Even though the balanced motors were mounted to ensure the same exposure to ambient air cooling, different working temperatures of $\approx 30^{\circ}$ for the left and $\approx 50^{\circ}$ for the right motor were observed. Such discrepancy suggests that an asymmetric motor loading existed along the course, which was not incorporated in the simulation where an average working temperature for a balanced motor model was taken at $\approx 40^{\circ}$.

Further evidence that the mathematical models of motorcycle and electric power train are reliable can be taken from figure 10. The left graph shows a linear trend between simulated battery pack current draw and motorcycle speed, which corresponds well with experimental observations. The same holds true for the battery pack current and the motor driving voltage on the right graph, as would be expected since motor speed and motor voltage are proportionally related for brushed DC motors.

Finally, figure 11 illustrates from simulation data the calculated overall efficiency of the complete energy conversion process: the mechanical power to ground is plotted as a function of the energy drained from the battery pack. The black lines correspond to overall efficiencies of $100 \%$ (solid) and $70 \%$ (dashed). The simulated motorcycle (blue dots) demonstrates an average overall efficiency close to or slightly below the $70 \%$ marker line.

\section{Motorbike optimisation}

The previous section has demonstrated the ability of the mathematical model to faithfully reproduce the entire run on the TT course, suggesting that simulation can now also be used to optimise the motorcycle design for the specific course. Lap time simulation can be effective in quickly highlighting performance variations, for example during selection of motorcycle configuration or race set-up parameters for which optimal values are not easily identified other than by experience or extensive historical data. In this section, we will demonstrate the value of optimisation via lap time simulation without such a priori 
knowledge during design and set-up alterations for three important electric motorcycle parameters: the wheel-to-motor gear ratio, the battery pack capacity, and the number of motors within the power train configuration.

The wheel-to-motor gear ratio mainly influences the motorcycle performance because it modifies both wheel torque and top speed. Since maximum motor torque and motor speed result from motor design and build and thus are fixed, a shorter wheel-to-motor gear ratio allows to reach higher wheel torque, and therefore faster motorcycle accelerations, yet at the same time it reduces maximum motorcycle speed. Moreover, figure 6 showed that, for a given motor power, the overall power train efficiency of motor, controller and battery pack increases as motor torque decreases, except for very low motor torques. On the basis of these considerations it is not immediately clear which gear ratio should be best used for the TT course. We therefore simulated different runs on the TT course varying the wheel-to-motor gear ratio in the range 2.5 to 4.3 , with step changes of 0.1 ; all the other characteristics of the motorcycle were kept unchanged. Results are shown in figure 12 and compared with reference values from the data and set-up of the (non-optimized) BX-15 chassis, as detailed in section 2.3. It can be noticed that the optimal lap time is approximately 1 minute and 45 seconds $(\approx 6 \%)$ less than the reference time, and it is obtained for a gear ratio of 3.4, which is significantly higher than the reference gear ratio 2.8. A lap time reduction of this magnitude corresponds to an increase in average motorcycle lap speed from $122 \mathrm{~km} / \mathrm{h}(75.8 \mathrm{mph})$ to $130 \mathrm{~km} / \mathrm{h}(80.8 \mathrm{mph})$. It can be observed that the optimal gear ratio provides neither maximum top speed ${ }^{1}$, nor maximum $0-120 \mathrm{~km} / \mathrm{h}$ acceleration. In fact, maximum top speed is achieved with a gear ratio equal to 3.1, while maximum acceleration is obtained with the shortest gear ratio considered (4.3). Figure 12 further shows that the gear ratio influences significantly the amount of energy used over the entire course (fourth graph from the top): the maximum energy usage (41MJ or $11.4 \mathrm{kWh}$ ) is obtained with a gear ratio equal to 3.3 , which is close to the optimal one, while the reference data shows approximately $10 \%$ less energy usage $(37 \mathrm{MJ}$ or $10.3 \mathrm{kWh})$ with gear ratio 2.8 . None of the gear ratios considered here would fully drain the battery pack; indeed the BX-15 battery pack size was intentionally overdimensioned on safety grounds to prevent full discharge. Moreover, the bottom graph in figure 12 shows that the overall efficiency, calculated as the total energy delivered to the wheel divided by the battery pack energy used, increases continuously with the gear ratio, at least in the range considered. The optimal gear ratio 3.4 uses slightly less battery pack energy than gear ratio 3.3 , and still allows to diminish the lap time thanks to a higher efficiency.

Motor current, voltage and motorcycle speed are compared in figure 13 for the reference and optimal gear ratios. As expected, the optimal gear ratio uses on average a higher motor driving voltage, but a lower motor current; in particular, with the optimal gear ratio the motor voltage is often equal to the maximum value above which motor damage could occur, while in the reference set-up such voltage values are never reached. The speed profile (bottom graph) shows that the optimal gear ratio provides higher accelerations but lower top speed than the reference set-up, corresponding to the discussion regarding figure 12 .

The second parameter under scrutiny is the battery pack capacity: an undersized battery pack will not provide enough power required by the motor, but an oversized battery pack would add extra weight to the motorcycle for the same energy usage. Sizing the pack capacity correctly is therefore again a critical but not straightforward design decision where simulation can help. We simulated several runs with varying battery pack

\footnotetext{
${ }^{1}$ It is the maximum speed achieved on the course, not the motorcycle top speed.
} 

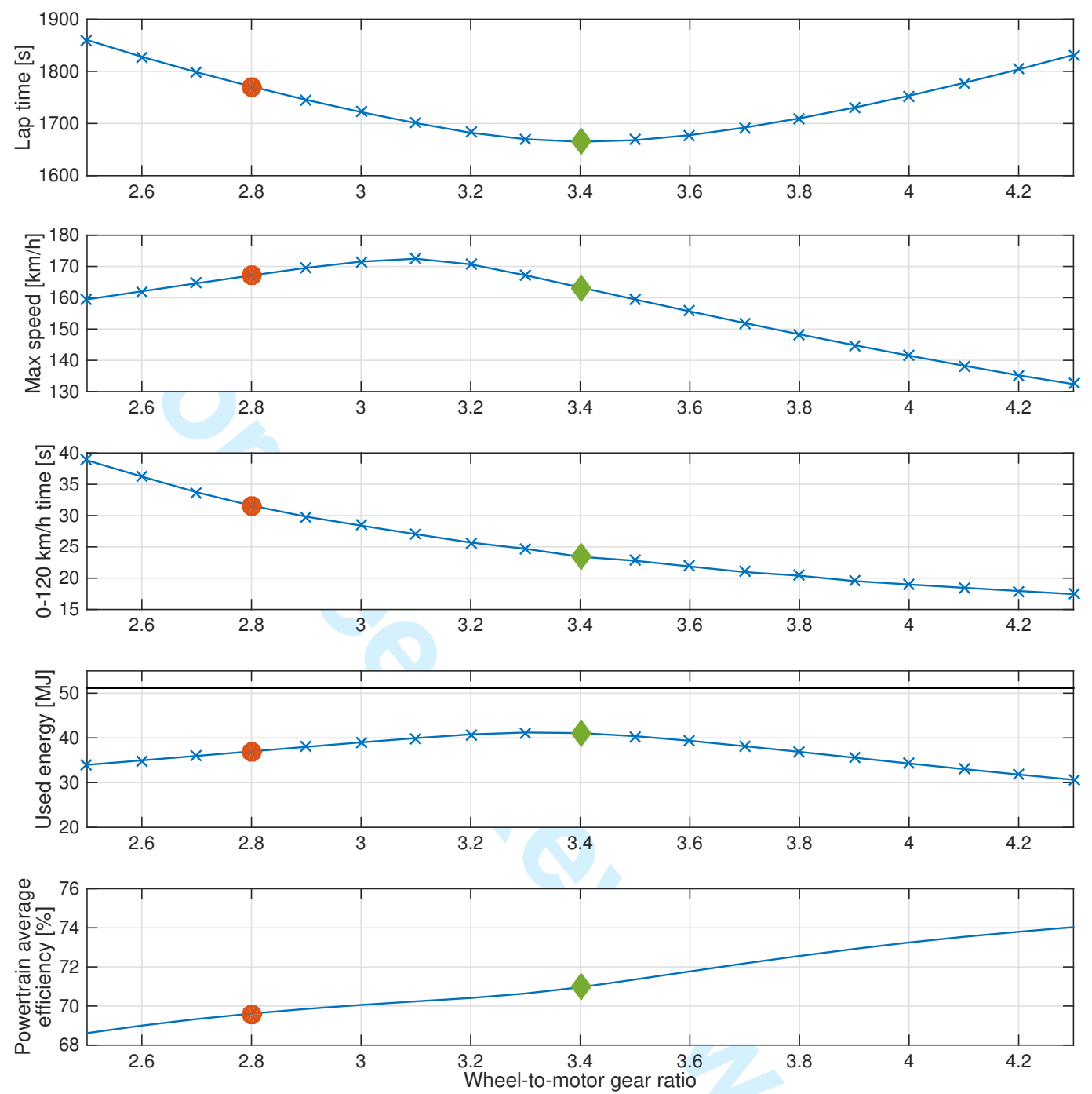

Figure 12.: From top to bottom, overall lap time, top speed, $0-120 \mathrm{~km} / \mathrm{h}$ acceleration time, energy used and overall power train efficiency are all shown vs. wheel-to-motor gear ratio. The red circle indicates the configuration used in the TT Zero Challenge, the green diamond indicates the optimal configuration as found through simulation. The black line in the fourth graph from the top represents total energy stored in the battery pack.

sizes and thus varying amounts of stored energy available, while keeping the energy density constant (at $0.51 \mathrm{MJ} / \mathrm{kg}$ or $142 \mathrm{Wh} / \mathrm{kg}$ as in the experimental reference set-up). The results obtained are illustrated in figure 14, where the top graph shows lap time and the bottom graph percentage energy used (e.g. 100\% means the battery pack has been completely drained). With the lowest battery capacity of $25 \mathrm{MJ}(6.9 \mathrm{kWh})$ considered, no energy is left at the end of the race and indeed to conclude the race it is necessary to adopt some energy saving strategies while navigating the course. As pack capacity increases, lap times reduce significantly and minimum lap time is reached for a capacity of (approximately) 37MJ $(10.3 \mathrm{kWh})$. Greater pack capacities are not necessary and the increment in weight reduces performance; however, the extra weight influences the lap 

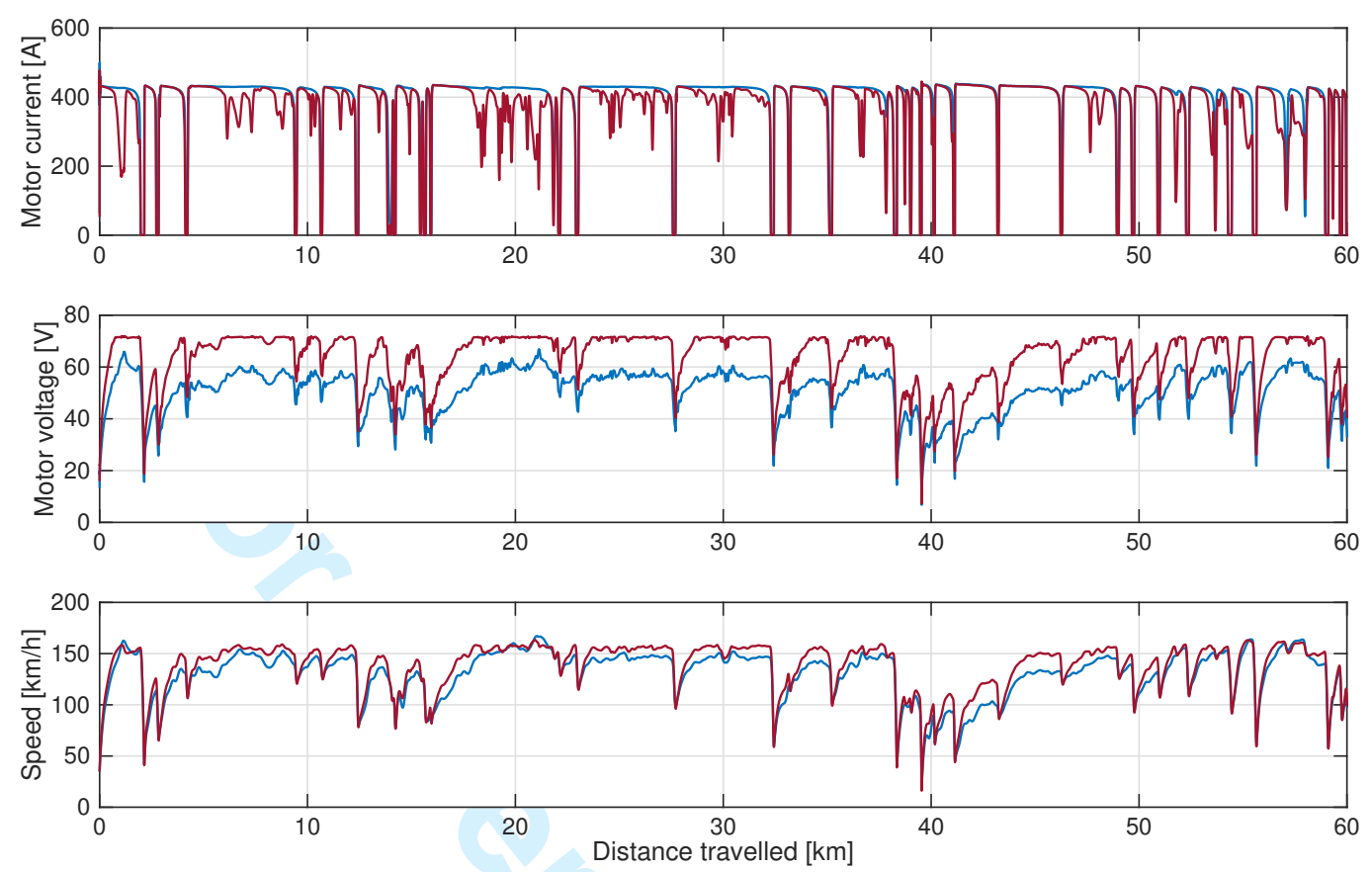

Figure 13.: Comparison between reference gear ratio 2.8 (blue lines) and optimised gear ratio 3.4 (magenta lines) for motor current, motor voltage and motorcycle speed.

time less significantly than energy saving strategies. This suggests that it is more convenient to provide the motorcycle with a slightly over-sized and heavier battery pack, than risking to be forced to adopt an energy conserving riding style.

The lap times shown in figure 14 are calculated with the reference gear ratio 2.8 , which is not optimal; moreover, we have previously seen that the gear ratio affects the battery usage (figure 12). Therefore, it is expected that the gear ratio and battery size should actually be optimised together. Figure 15 shows lap time as a function of both gear ratio and battery capacity. A lap time of 27 minutes and 34 seconds (nearly two minutes or $\approx 6.5 \%$ faster than the reference configuration) may be obtained with a gear ratio of 3.4 and a battery pack capacity of $\approx 42 \mathrm{MJ}(11.7 \mathrm{kWh})$, corresponding to an increase in average motorcycle lap speed from $122 \mathrm{~km} / \mathrm{h}(75.8 \mathrm{mph})$ to $131 \mathrm{~km} / \mathrm{h}(81.4 \mathrm{mph})$. The contour graph highlights that the lap time significantly increases when the chosen gear ratio is far from the optimal one (i.e. greater than 3.6 or lower than 3.3) or when the battery capacity is under-sized (i.e. less than $37 M J$ or $10.3 \mathrm{kWh}$ ). Moreover, figure 15 confirms what was already observed in figure 14, namely that an over-sized battery pack leads to a slight performance decrease, but an under-sized battery pack causes a significant increase of the lap time. By the way the figure shows also that the gear ratio and the battery capacity optimization are almost independent.

The third parameter that has been optimised here through lap time simulation is the number of motors in the power train. BX-15 was equipped with a dual motor configuration, a popular choice because of relative ease of packaging and obvious advantage over a single motor of the same specification. Considering that the performance of the motorcycle studied in this work is limited mainly by motor power, adding one motor seems a worthwhile consideration at first. However one more motor would also add $11 \mathrm{~kg}$ of extra weight, and it would require a bigger battery pack. For this reason it cannot be 

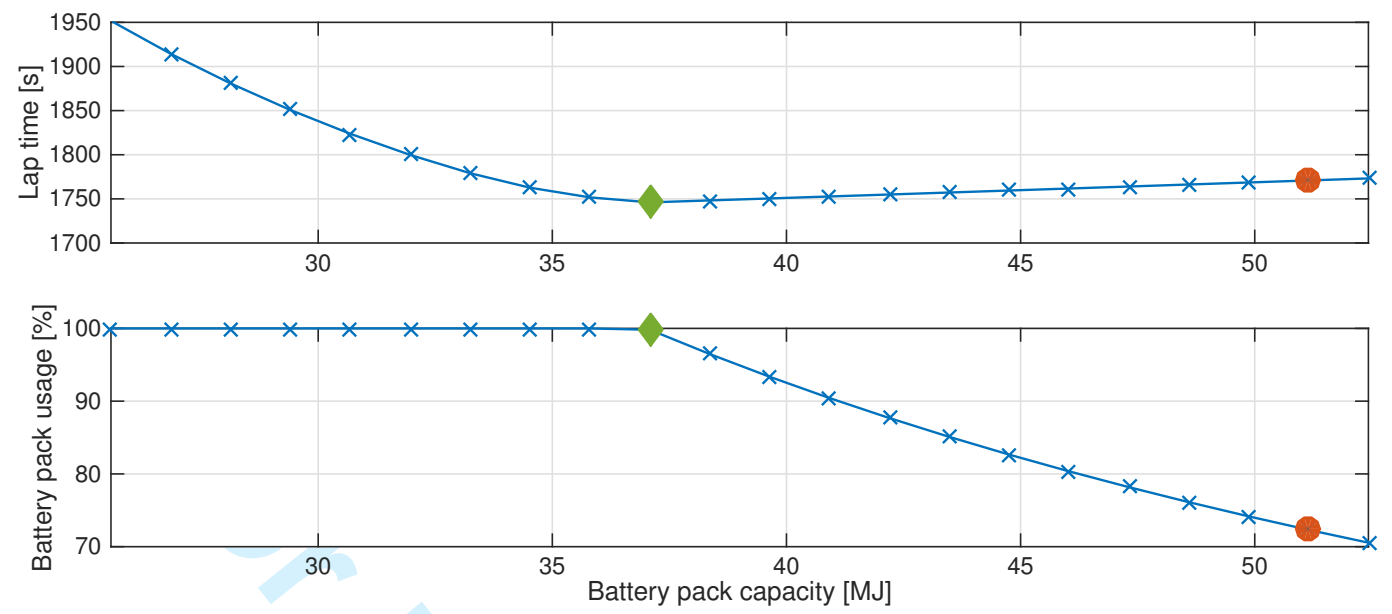

Figure 14.: Overall lap time (top) and battery usage percentage (bottom) are shown vs. the total amount of energy stored in the battery pack (energy density held constant at reference value $0.51 \mathrm{MJ} / \mathrm{kg}$ or $142 \mathrm{Wh} / \mathrm{kg}$ ). The red circle indicates the configuration used in the TT Zero Challenge, the green diamond indicates the optimal configuration as found through simulation.

clear a priori whether two or three motors would constitute the best configuration, and simulation can again provide clarity.

We simulated the lap time on the TT course for a motorcycle with a triple motor configuration, with no other changes applied; total mass was increased by $11 \mathrm{~kg}$ (weight of one motor) but the reference gear ratio and battery pack capacity were kept unchanged. The simulated lap time (26 minutes and 6 seconds or $1566 \mathrm{~s}$, as reported in table 1) turned out to be significantly lower than that of the dual motor configuration (even of the optimised version). But the simulation showed also that with three motors the $4 \mathrm{P} 24 \mathrm{~S}$ battery pack is being fully discharged, suggesting that the performance could be increased by parallel mounting an extra battery sequence. However, in 5P24S pack arrangement it turns out that the lap time becomes five seconds more (1571s, see table 1), therefore the additional energy provided by the extra battery capacity does not compensate for the increased motorcycle weight. In conclusion, a three motor configuration is the best choice in terms of minimum lap time because it allows to increase the average speed on the TT Course by the $\approx 14 \%$ from $122 \mathrm{~km} / \mathrm{h}(75.8 \mathrm{mph})$ to $138 \mathrm{~km} / \mathrm{h}(85.7 \mathrm{mph})$ (with the reference gear ratio). However, these results should be considered against the actual design and build process, since a triple motor configuration is more difficult to package and integrate into the motorcycle chassis than the dual motor configuration.

\begin{tabular}{|c|c|c|c|}
\hline & $\begin{array}{c}2 \text { motors \& 4P24S } \\
(51 \mathrm{MJ}-14.2 \mathrm{kWh})\end{array}$ & $\begin{array}{c}3 \text { motors \& 4P24S } \\
(51 \mathrm{MJ}-14.2 \mathrm{kWh})\end{array}$ & $\begin{array}{c}3 \text { motors \& 5P24S } \\
(64 \mathrm{MJ}-17.8 \mathrm{kWh})\end{array}$ \\
\hline Lap time & $29 \mathrm{~m} 31 \mathrm{~s}$ & $26 \mathrm{~m} \mathrm{~s}$ & $26 \mathrm{~m} \mathrm{11s}$ \\
\hline Battery usage & $72 \%$ & $100 \%$ & $85 \%$ \\
\hline
\end{tabular}

Table 1.: Comparison of lap times with different motor configurations and battery pack arrangements. 


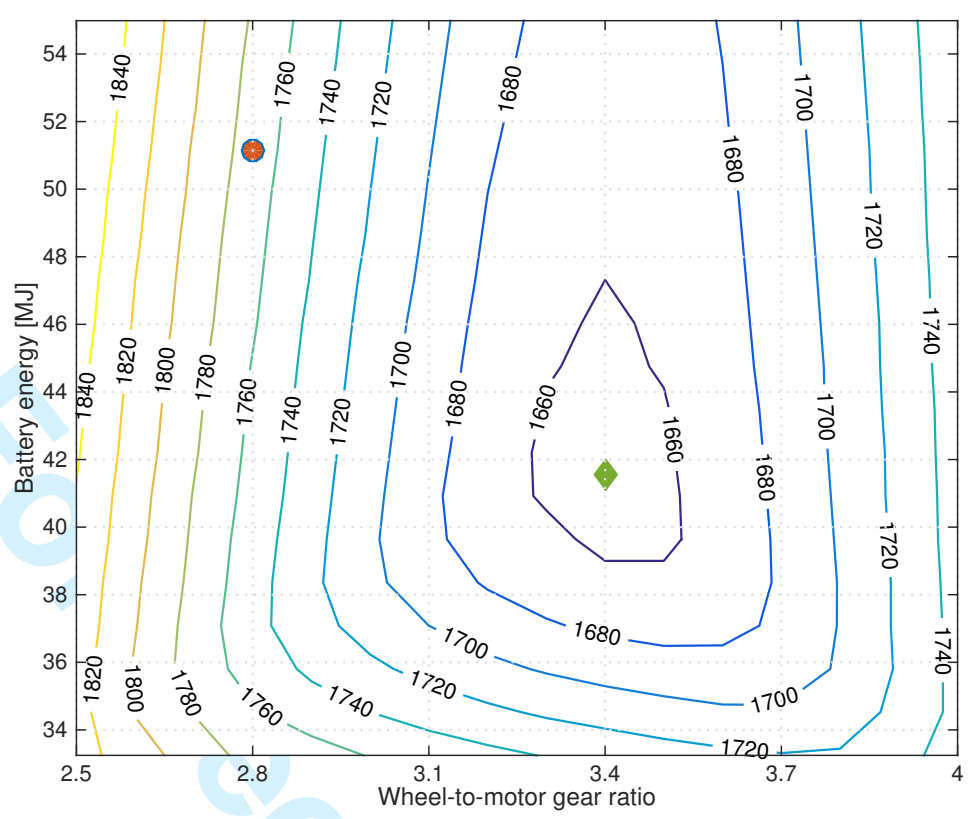

Figure 15.: Lap time contour graph as a function of gear ratio and battery pack capacity. The minimum lap time of 27 minutes and 34 seconds (1654s) is obtained with a gear ratio of 3.4 and a battery pack capacity of $\approx 42 \mathrm{MJ}(11.7 \mathrm{kWh})$. The red circle indicates the configuration used in the TT Zero Challenge, the green diamond indicates the optimal configuration as found through simulation.

\section{Conclusions}

Electric and hybrid vehicles are nowadays rapidly gaining in popularity, but performance and energy consumption optimisation remain a challenging task. In this work we used an optimal control lap time model to simulate an electric motorcycle for the TT Zero Challenge over the entire 60km-long (37.73mi) Snaefell Mountain Course on the Isle of Man. A simple motorcycle multibody model, able to capture the essence of the dynamics, has been used in order to efficiently run simulations over such a long road. A threedimensional road model and an electric power train model, consisting of battery pack, DC brushed motors and motor controller, have been implemented. The model allows to efficiently perform simulations over the $60 \mathrm{~km}$ road course in less than three minutes, and the simulation outcomes have been validated through comparison with experimental data acquired by the Brunel Racing team during the 2015 TT Zero event.

Lap time simulation was then used to optimise the design of the racing motorcycle, in particular the wheel-to-motor gear ratio, the battery pack capacity and the number of motors in the power train. Results showed that the gear ratio has a significant influence on the performance and on the efficiency of the power train, with the use of the optimal (shorter) gear ratio leading to a lap time reduction of approximately 2 minutes or $6 \%$ of the reference experimental data. Perhaps surprisingly, battery pack capacity turned out to affect lap time only marginally, as long as the pack is sized or over-sized so as not to be fully discharged over one lap. However, when battery pack capacity is undersized, such that energy saving riding strategies must be adopted in order to complete the entire course, then lap time increases significantly as the reduction in motorcycle weight does not overcome the lack of energy to ride at full performance throughout. Finally, 
the optimisation of the multiple motor configuration demonstrated that adopting an extra motor and upgrading to a triple (from dual) configuration brings along additional performance improvement: the extra available power reduces lap time and overcomes the increase in weight. These results suggest that the bespoke developed optimal control model for lap time simulation can serve as a valuable tool in the motorcycle design process, because of the sensitivity it harnesses in motorcycle parameter variation studies and the relatively short computing time that is required.

\section{Appendix A. Motorbike main characteristics}

\begin{tabular}{|c|c|c|c|}
\hline symbol & value & units & description \\
\hline$g$ & 9.81 & $\mathrm{~m} / \mathrm{s}^{2}$ & gravitational acceleration \\
\hline$\rho$ & 1.2 & $\mathrm{~kg} / \mathrm{m}^{3}$ & air density \\
\hline$h$ & 0.6 & $\mathrm{~m}$ & centre of gravity ( $\mathrm{CoG}$ ) height \\
\hline$r$ & 0.1 & $\mathrm{~m}$ & wheel radius \\
\hline$r_{t}$ & 0.3 & $\mathrm{~m}$ & wheel toroid radius \\
\hline$M$ & 290 & $\mathrm{~kg}$ & motorbike mass including rider and 4 battery packs \\
\hline$I_{x x}$ & 19 & $\mathrm{kgm}^{2}$ & motorbike roll moment of inertia \\
\hline$I_{g y}$ & 2.1 & $\mathrm{kgm}^{2}$ & gyroscopic moment of inertia \\
\hline$I_{w y}$ & 0.7 & $\mathrm{kgm}^{2}$ & wheel spin moment of inertia \\
\hline$K_{\beta}$ & 12 & & tyre sideslip stiffness \\
\hline$K_{\phi}$ & 1 & & tyre roll stiffness \\
\hline$K_{s}$ & $2 \mathrm{e} 5$ & $\mathrm{~N} / \mathrm{m}$ & tyre radial stiffness \\
\hline$K_{c}$ & $1 \mathrm{e} 3$ & $\mathrm{Ns} / \mathrm{m}$ & tyre radial damping \\
\hline$\mu_{x}$ & 0.8 & & tyre longitudinal adherence \\
\hline$\mu_{y}$ & 0.6 & & tyre lateral adherence \\
\hline$\mu_{r}$ & 0.015 & & tyre rolling resistance \\
\hline$C_{d}$ & 0.41 & $\mathrm{~m}^{2}$ & drag coefficient \\
\hline$C_{l}$ & 0.03 & $\mathrm{~m}^{2}$ & lift coefficient \\
\hline
\end{tabular}

Table A1.: Vehicle parameters.

\section{References}

[1] Zainab Asus, El-Hassane Aglzim, Daniela Chrenko, Zul-Hilmi Che Daud, and Luis Le Moyne. Dynamic modeling and driving cycle prediction for a racing series hybrid car. IEEE Journal of Emerging and Selected Topics in Power Electronics, 2(3):541-551, 2014.

[2] Enrico Bertolazzi, Francesco Biral, and Mauro Da Lio. Symbolic-numeric indirect method for solving optimal control problems for large multibody systems. Multibody System Dynamics, 13(2):233-252, 2005.

[3] Enrico Bertolazzi, Francesco Biral, and Mauro Da Lio. Symbolic-numeric efficient solution of optimal control problems for multibody systems. Journal of computational and applied mathematics, 185(2):404-421, 2006. 
[4] John T Betts. Practical methods for optimal control and estimation using nonlinear programming, volume 19. Siam, 2010.

[5] Francesco Biral, Enrico Bertolazzi, and Paolo Bosetti. Notes on numerical methods for solving optimal control problems. IEEJ Journal of Industry Applications, 5(2):154-166, 2016.

[6] Arthur Earl Bryson. Dynamic optimization. Addison Wesley Longman Menlo Park, CA, 1999.

[7] Daniele Casanova. On minimum time vehicle manoeuvring: The theoretical optimal lap. 2000.

[8] Daniele Casanova, Robin S Sharp, and Pat Symonds. Minimum time manoeuvring: The significance of yaw inertia. Vehicle system dynamics, 34(2):77-115, 2000.

[9] Daniele Casanova, Robin S Sharp, and Pat Symonds. On the optimisation of the longitudinal location of the mass centre of a formula one car for two circuits. In Proceedings of AVEC, volume 2, pages $6-12,2002$.

[10] V Cossalter, M Peretto, and S Bobbo. Investigation of the influences of tyre - road friction and engine power on motorcycle racing performance by means of the optimal manoeuvre method. Proceedings of the Institution of Mechanical Engineers, Part D: Journal of Automobile Engineering, 224(4):503$519,2010$.

[11] Vittore Cossalter, Mauro Da Lio, Roberto Lot, and Lucca Fabbri. A general method for the evaluation of vehicle manoeuvrability with special emphasis on motorcycles. Vehicle system dynamics, 31(2):113-135, 1999.

[12] Vittore Cossalter, Roberto Lot, and Davide Tavernini. Optimization of the centre of mass position of a racing motorcycle in dry and wet track by means of the "optimal maneuver method". In Mechatronics (ICM), 2013 IEEE International Conference on, pages 412-417. IEEE, 2013.

[13] DA Crolla, Q Ren, S ElDemerdash, and F Yu. Controller design for hybrid vehicles - state of the art review. In 2008 IEEE Vehicle Power and Propulsion Conference, pages 1-6. IEEE, 2008.

[14] Jacques Denavit. A kinematic notation for lower-pair mechanisms based on matrices. Trans. of the ASME. Journal of Applied Mechanics, 22:215-221, 1955.

[15] Lino Guzzella, Antonio Sciarretta, et al. Vehicle propulsion systems, volume 1. Springer, 2007.

[16] Austin Hughes and Bill Drury. Electric motors and drives: fundamentals, types and applications. Newnes, 2013.

[17] Daniel Patrick Kelly. Lap time simulation with transient vehicle and tyre dynamics. 2008.

[18] Daniel Patrick Kelly and Robin S Sharp. Time-optimal control of the race car: influence of a thermodynamic tyre model. Vehicle System Dynamics, 50(4):641-662, 2012.

[19] Stefan Koehler, Alexander Viehl, Oliver Bringmann, and Wolfgang Rosenstiel. Improved energy efficiency and vehicle dynamics for battery electric vehicles through torque vectoring control. In 2015 IEEE Intelligent Vehicles Symposium (IV), pages 749-754. IEEE, 2015.

[20] David Limebeer. Optimising the aero-suspension interactions in a forumla one car. IEEE Transactions on Control Systems Technology, 82014.

[21] David JN Limebeer. Optimal control of a formula one car on a three-dimensional track - part 1: Track modeling and identification. Journal of Dynamic Systems, Measurement, and Control, 137(5):1-11, 2015.

[22] David JN Limebeer, Giacomo Perantoni, and AV Rao. Optimal control of formula one car energy recovery systems. International Journal of Control, 87(10):2065-2080, 2014.

[23] R Lot and M Da Lio. A symbolic approach for automatic generation of the equations of motion of multibody systems. Multibody System Dynamics, 12(2):147-172, 2004.

[24] Roberto Lot and Francesco Biral. A curvilinear abscissa approach for the lap time optimization of racing vehicles. IFAC Proceedings Volumes, 47(3):7559-7565, 2014.

[25] Roberto Lot and Nicola Dal Bianco. Lap time optimisation of a racing go-kart. Vehicle System Dynamics, pages 1-21, 2015.

[26] Roberto Lot and Nicola Dal Bianco. The significance of high-order dynamics in lap time simulations. In 24th International Symposium on Dynamics of Vehicles on Road and Tracks, 2015.

[27] Roberto Lot and S Evangelou. Lap time optimization of a sports series hybrid electric vehicle. In 2013 World Congress on Engineering, 2013.

[28] Giacomo Perantoni and David JN Limebeer. Optimal control for a formula one car with variable parameters. Vehicle System Dynamics, 52(5):653-678, 2014.

[29] Laura V Pérez and Elvio A Pilotta. Optimal power split in a hybrid electric vehicle using direct transcription of an optimal control problem. Mathematics and Computers in Simulation, 79(6):19591970, 2009.

[30] Enid R Pinch. Optimal control and the calculus of variations. Oxford University Press Oxford, 1993.

[31] Lennon Rodgers, Radu Gogoana, Thomas German, Mark Jeunnette, Randall Briggs, Erick Fuentes, and Will Pritchett. Designing an electric motorcycle for the isle of man tt zero race, and how electric 
vehicle racing could be used to spur innovation. In EVS26 International Battery, Hybrid and Fuel Cell Electric Vehicle Symposium, 2012.

[32] Farzad Rajaei Salmasi. Control strategies for hybrid electric vehicles: Evolution, classification, comparison, and future trends. IEEE Transactions on vehicular technology, 56(5):2393-2404, 2007.

[33] RS Sharp. A method for predicting minimum-time capability of a motorcycle on a racing circuit. Journal of Dynamic Systems, Measurement, and Control, 136(4):041007, 2014.

[34] Stephanie Stockar, Vincenzo Marano, Marcello Canova, Giorgio Rizzoni, and Lino Guzzella. Energyoptimal control of plug-in hybrid electric vehicles for real-world driving cycles. IEEE Transactions on Vehicular Technology, 60(7):2949-2962, 2011.

[35] Davide Tavernini, Matteo Massaro, Efstathios Velenis, Diomidis I Katzourakis, and Roberto Lot. Minimum time cornering: the effect of road surface and car transmission layout. Vehicle System Dynamics, 51(10):1533-1547, 2013.

[36] Davide Tavernini, Efstathios Velenis, Roberto Lot, and Matteo Massaro. The optimality of the handbrake cornering technique. Journal of Dynamic Systems, Measurement, and Control, 136(4):041019, 2014.

[37] AJ Tremlett and DJN Limebeer. Optimal tyre usage for a formula one car. Vehicle System Dynamics, 54(10):1448-1473, 2016.

[38] Joerg Dieter Weigl, Martin Henz, Hamdani Saidi, et al. Converted battery-powered electric motorcycle and hydrogen fuel cell-powered electric motorcycle in south east asia: Development and performance test. In Proceedings of the Joint International Conference on Electric Vehicular Technology and Industrial, Mechanical, Electrical and Chemical Engineering (ICEVT 8 IMECE), pages 1-4. IEEE, 2015.

[39] Joerg Dieter Weigl, Hamdani Saidi, et al. Design, testing and optimisation of a hydrogen fuel cell motorcycle for south east asia. In Ecological Vehicles and Renewable Energies (EVER), 2013 8th International Conference and Exhibition on, pages 1-4. IEEE, 2013. 